\title{
Salt-sensitive hypertension in circadian clock-deficient Cry-null mice involves dysregulated adrenal Hsd3b6
}

Masao Doi ${ }^{1}$, Yukari Takahashi ${ }^{1}$, Rie Komatsu ${ }^{1}$, Fumiyoshi Yamazaki ${ }^{1}$, Hiroyuki Yamada ${ }^{1}$, Shogo Haraguchi $^{2}$, Noriaki Emoto ${ }^{3}$, Yasushi Okuno ${ }^{4}$, Gozoh Tsujimoto ${ }^{5}$, Akihiro Kanematsu ${ }^{6}$, Osamu Ogawa ${ }^{6}$, Takeshi Todo ${ }^{7}$, Kazuyoshi Tsutsui ${ }^{2}$, Gijsbertus T J van der Horst $^{8}$ \& Hitoshi Okamura ${ }^{1}$

${ }^{1}$ Department of Systems Biology, Graduate School of Pharmaceutical Sciences, Kyoto University, Sakyo-ku, Kyoto, Japan. ${ }^{2}$ Laboratory of Integrative Brain Sciences, Department of Biology, Faculty of Education and Integrated Arts and Sciences, Waseda University, Shinjuku-ku, Tokyo, Japan. ${ }^{3}$ Division of Cardiovascular and Respiratory Medicine, Department of Internal Medicine, Kobe University Graduate School of Medicine, Chuo-ku, Kobe, Japan. ${ }^{4}$ Department of Systems Bioscience for Drug Discovery and ${ }^{5}$ Department of Genomic Drug Discovery Science, Graduate School of Pharmaceutical Sciences, ${ }^{6}$ Department of Urology, Graduate School of Medicine, Kyoto University, Sakyo-ku, Kyoto, Japan. ${ }^{7}$ Department of Radiation Biology and Medical Genetics, Graduate School of Medicine, Osaka University, Yamadaoka, Suita, Japan. ${ }^{8}$ Department of Genetics, Center for Biomedical Genetics, Erasmus University Medical Center, Rotterdam, The Netherlands.

Correspondence should be addressed to H.O. (okamurah@pharm.kyoto-u.ac.jp).

Malfunction of the circadian clock has been linked to the pathogenesis of a variety of diseases. We show that mice lacking the core clock components Cryptochrome 1 (Cry1) and Cryptochrome 2 (Cry2) (Cry-null mice) show salt-sensitive hypertension due to abnormally high synthesis of the mineralocorticoid aldosterone by the adrenal gland. An extensive search for the underlying cause led us to identify type VI $3 \beta$-hydroxyl-steroid dehydrogenase (Hsd3b6) as a new hypertension risk factor in mice. Hsd3b6 is expressed exclusively in aldosterone-producing cells and is under transcriptional control of the 
circadian clock. In Cry-null mice, $\mathrm{Hsd} 3 \mathrm{b6}$ messenger RNA and protein levels are constitutively high, leading to a marked increase in $3 \beta$-hydroxysteroid

dehydrogenase-isomerase (3 $\beta$-HSD) enzymatic activity and, as a consequence, enhanced aldosterone production. These data place Hsd3b6 in a pivotal position through which circadian clock malfunction is coupled to the development of hypertension. Translation of this finding to humans will need clinical examination of human HSD3B1 gene, which we found to be functionally similar to mouse $\mathrm{Hsd} 3 \mathrm{~b} 6$

Hypertension is a complex trait, influenced by multiple genetic and environmental factors ${ }^{1}$. Although it has been estimated that approximately $30-50 \%$ of blood pressure variance is due to inherited genes, the molecular basis of this disease remains largely to be defined ${ }^{2}$. In mammals, behavior, physiology and metabolism are subject to a well-controlled daily rhythm, generated by an internal self-sustained molecular oscillator referred to as the circadian clock ${ }^{3-7}$. Because up to $10 \%$ of the transcriptome has been estimated to be under the control of the circadian $\operatorname{clock}^{8}$, it may not come as a surprise that malfunction of this time keeper can result in the onset of a variety of pathological events ${ }^{9,10}$. Indeed, epidemiological studies show that shift workers, long-distance transmeridian flight crews and individuals with sleep disorders show a higher than average prevalence of cardiovascular diseases ${ }^{11-13}$. Thus, it has been speculated that impediment of the circadian clock may underlie altered cardiovascular homeostasis. However, the molecular mechanisms by which circadian clock malfunction contribute to cardiovascular disease, including hypertension, are poorly understood.

Cry-null mice lack a functional circadian clock and accordingly show arrhythmic behavior, physiology and metabolism ${ }^{14-16}$. Cry proteins act as potent transcriptional repressors that downregulate transcription of E-box (CACGTG) enhancer-containing clock genes (including the Period- and Cryptochrome-encoding genes), as well as a wide variety of 
clock-controlled genes (including circadian transactivator Dbp-encoding gene) ${ }^{16-18}$. Time of day-dependent activation and repression of such E-box-containing genes are central features of normal circadian clock function. In Cry-null mice, the expression of E-box-containing genes is constitutively increased (derepressed) as a result of impaired circadian repression $^{16,19,20}$.

To date, little is known about the pathological states of Cry-null mice. We and others have focused on the adrenal gland in investigating how the circadian clock system affects metabolism ${ }^{21,22}$. In characterizing the endocrinological status of Cry-deficiency mice, we found abnormally high levels of aldosterone, a steroid hormone that is secreted by the adrenal glands. Aldosterone acts as a potent mineralocorticoid that promotes sodium and water retention by the kidney, thereby increasing vascular fluid volume and blood pressure ${ }^{23,24}$. The plasma aldosterone concentration (PAC) is finely controlled by the renin-angiotensin-aldosterone system (RAAS), which regulates cardiovascular function and blood pressure homeostasis ${ }^{2}$. Because abnormal elevation of PAC has been linked to the pathogenesis of human hypertension ${ }^{23-25}$, our observation of increased PAC in Cry-null mice prompted us to investigate how the circadian clock could be linked to the etiology of aldosterone-dependent disorders.

Here we show that Cry-null mice have salt-sensitive hypertension due to the abnormal production of aldosterone by the adrenal glands. An extensive search for a steroidogenic enzyme that causes the adrenal dysfunction in Cry-null mice led us to identify Hsd3b6 as a key enzyme through which Cry gene inactivation results in an abnormally high PAC and salt-sensitive hypertension. Furthermore, we show that the human HSD3B1 gene is a functional counterpart to the mouse Hsd3b6 gene, as HSD3B1 is expressed in aldosterone-producing cells in the human adrenal gland. 


\section{RESULTS}

\section{Chronic hyperproduction of aldosterone by Cry-null adrenal glands}

After our initial observation of increased PAC in Cry-null mice, we quantified PAC and plasma renin activity (PRA) in wild-type (WT) and mutant mice kept in constant darkness and sampled at selected circadian time points (Fig. 1). Cry-null mice showed abnormally high PAC throughout the day (Fig. 1a). In line with previous reports in rodents ${ }^{26}$, WT mice showed a mild circadian fluctuation in PAC (Fig. 1a) with overall values lower than those in Cry-null mice. The elevated PAC in Cry-null mice was not a secondary result of enhanced PRA, as PRA was markedly suppressed in Cry-null mice at both CT0 and CT12 (being defined as the beginning of subjective day and night, respectively) (Fig. 1b). The decreased PRA in Cry-null mice is most likely due to RAAS-dependent feedback inhibition resulting from the elevated PAC ${ }^{23,24}$.

The increased PAC despite a marked reduction of PRA suggested that the functioning of the adrenal gland in Cry-null mice might be affected. To assess the functionality of the Cry-null adrenal gland, we used an ex vivo tissue culture system that allowed us to evaluate the rate of aldosterone production by measuring the amount of aldosterone secreted into the culture medium. We found that the aldosterone production rate of the Cry-null adrenal gland was significantly higher than that of the WT adrenal gland (Fig. 1c). This increased aldosterone production was observed in both adrenal glands of a Cry-null mouse (Supplementary Fig. 1). Taken together, these data provide evidence that Cry-null mice have a profoundly increased PAC as a result of enhanced aldosterone secretion by the adrenal gland.

\section{Chronic hyperexpression of Hsd3b6 in Cry-null adrenal glands}

To dissect the altered steroidogenesis in Cry-null mice at the molecular level, we used microarrays to survey all known mouse steroidogenic genes for their expression in the adrenal 
gland (Fig. 2a). This survey identified a gene probe (1460232_s_at) for which signal intensities in the Cry-null adrenal gland were markedly increased throughout the day as compared to the WT gland. This gene probe potentially hybridizes with the Hsd3b2, Hsd3b3 and Hsd3b6 mRNA isoforms of the Hsd3b gene family. These isoforms are known to encode

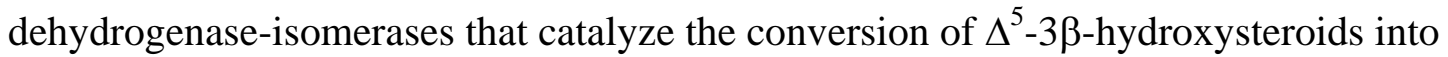
hormonally active $\Delta^{4}$-3-ketosteroids ${ }^{27}$, an enzymatic reaction required for aldosterone biosynthesis ${ }^{28}$.

To identify all $H s d 3 b$ isoforms expressed in the mouse adrenal gland, we used an RT-PCR-based assay in combination with restriction endonuclease-mapping analysis (Supplementary Methods). We subjected adrenal total RNA to RT-PCR with a primer set capable of amplifying all classes of mouse $H s d 3 b$ genes (types I to VI). We then analyzed the identity of the PCR products by digestion of the DNA with subtype-specific restriction endonucleases (Supplementary Table 1). We observed that mouse adrenal glands express only type I and VI Hsd3b isoforms; the other isoforms (types II to V) were undetectable (Supplementary Fig. 2). Quantitative RT-PCR (qRT-PCR) analysis with gene-specific primers further showed that the $H s d 3 b$ gene aberrantly expressed in Cry-null adrenal glands was Hsd3b6 rather than Hsd3b1; that Hsd3b6 mRNA levels in Cry-null adrenal glands were constitutively increased across circadian time; and that Hsd3b6 expression in WT adrenal glands, although at relatively low levels, was subject to circadian fluctuation (Fig. 2b). In vitro promoter analysis (Supplementary Fig. 3a) revealed that transcription of Hsd3b6 is positively controlled by Dbp, suggesting that the enhanced Dbp expression in Cry-null mice (refs 17, 20. and Supplementary Fig. 3b) could account for the hyperexpression of Hsd3b6 in Cry-null adrenal glands (Supplementary Fig. 3c,d) 


\section{Hsd3b6 is exclusively expressed in zona glomerulosa cells}

In situ hybridization analysis with a radiolabeled $H s d 3 b 6$ probe (Fig. 2c) not only confirmed the marked elevation of Hsd3b6 mRNA in Cry-null adrenal glands but also uncovered a unique spatial arrangement of Hsd3b6 expression. This analysis showed that Hsd3b6 is expressed almost exclusively in the outer layer of the adrenal cortex (Fig. 2c and Supplementary Fig. 4). Analysis of Hsd3b6 expression by emulsion autoradiography (data not shown) and digoxigenin in situ hybridization (Supplementary Fig. 5a) further illustrated that the Hsd3b6-positive cells were localized in the outermost cortical layer, termed the zona glomerulosa.

The predominant expression of Hsd3b6 in the zona glomerulosa is particularly noteworthy because the production of aldosterone is known to take place exclusively in this region $^{29}$. The zona glomerulosa is functionally characterized by the expression of the Cyp11b2 gene, which encodes the aldosterone synthase required for the final step of aldosterone synthesis $^{30,31}$. Notably, our in situ hybridization analysis suggested that Hsd3b6 and Cyp11b2 are both expressed mainly in adrenal zona glomerulosa cells (Fig. 2c and Supplementary Fig. 5b). In marked contrast to the local expression of $H s d 3 b 6, H s d 3 b 1$ was expressed more broadly in the adrenal cortex (Fig. 2c), similar to expression of the Cyp11b1 gene, which encodes the steroid $11 \beta$-hydroxylase involved in the production of corticosterone ${ }^{30,32}$. These data thus disclose a previously uncharacterized spatial and functional separation between Hsd3b6 and Hsd3b1 in the mouse adrenal gland.

To further confirm the local expression of Hsd3b6 in zona glomerulosa cells, we generated an antibody to Hsd3b6 and performed immunohistochemistry (Fig. 3). Notably, almost all zona glomerulosa cells in the Cry-null adrenal gland were found to be immunoreactive to the Hsd3b6-specific antibody (Fig. 3a). To determine whether Cyp11b2 is coexpressed with Hsd3b6, we further processed immunostained adrenal sections for in situ 
hybridization with a digoxigenin-labeled Cyp11b2 probe. This double-labeling analysis revealed that nearly all Hsd3b6-positive cells also expressed Cyp11b2 (Fig. 3b). The converse was also true; almost all Cyp11b2-positive cells were Hsd3b6-immunopositive (Fig. 3b). This high degree of colocalization of Hsd3b6 protein with the aldosterone synthase Cyp11b2 mRNA strongly suggests that the zona glomerulosa-specific Hsd3b6 isoform is involved in aldosterone production in the mouse adrenal gland.

\section{Steroidogenic genes in Cry-null zona glomerulosa cells}

As the zona glomerulosa cell population constitutes only a fraction of the total number of adrenal gland cells, assays using whole adrenal gland (Supplementary Fig. 2) could lead to an underestimation of Hsd3b6 expression in this tissue. Indeed, probably because of this reason, the presence of adrenal Hsd3b6 has been left undefined to date ${ }^{27,28}$. We next carried out laser microdissection to examine in more detail the zonal expression of Hsd3b6 and Hsd3b1 in the WT adrenal gland. (Fig. 2d). Cells in either the zona glomerulosa or the zona fasciculata (a middle layer of the adrenal cortex) were separately excised by laser microdissection; total RNA extracts from these cells were then analyzed by RT-PCR with a primer set that can amplify both $H s d 3 b 1$ and Hsd3b6 (the sequence similarity between these two genes enabled amplification of both genes with a common primer set). Subtype-specific DNA digestion revealed almost equivalent concentrations of $H s d 3 b 1$ and $H s d 3 b 6$ mRNA in the zona glomerulosa cells (Fig. 2d), suggestive of a substantial role of Hsd3b6 in aldosterone production by WT adrenal glands. Of note, Hsd3b6 was zona glomerulosa specific, with virtually no expression in zona fasciculata cells, whereas Hsd3b1 was predominantly expressed in zona fasciculata cells (Fig. 2d). A similar examination of subtype-specific expression of Cyp11b genes showed that, like Hsd3b6, expression of Cyp11b2 was confined to zona glomerulosa cells (Fig. 2d and Supplementary Table 2). These observations confirm the cellular purity of our laser 
microdissection samples and provide further evidence of the colocalization of Hsd3b6 and Cyp11b2 expression in zona glomerulosa cells.

We also performed qRT-PCR analysis on zona glomerulosa cell-specific RNA preparations and confirmed that the Hsd3b6 mRNA level was drastically increased in Cry-null zona glomerulosa cells as compared to WT cells (Fig. 2e). We did not observe such elevated expression for transcripts of other steroidogenic genes tested (Star (encoding steroidogenic acute regulatory protein), Cyp11a1, Hsd3b1, Cyp21a1, Cyp11b1 and Cyp11b2) (Fig. 2e).

\section{Chronically enhanced 3ß-HSD activity in Cry-null mice}

If the observed increase in the levels of $H s d 3 b 6$ mRNA is functionally relevant, it should be accompanied by increased accumulation of Hsd3b6 protein in Cry-null adrenal glands. Immunoblot analysis revealed that the amount of Hsd3b6 protein in the Cry-null adrenal gland was highly elevated at both CT0 and CT12, whereas the amount of Cyp11a1 protein remained unchanged (Fig. 4a). Next, we assayed 3ß-HSD enzymatic activity in WT and Cry-null enucleated adrenal glands (that is, the capsular portion of the glands), which consist mainly of zona glomerulosa cells. At both CT0 and CT12, we observed a marked increase of 3 $\beta$-HSD activity in enucleated adrenal glands from Cry-null mice compared to WT mice, regardless of

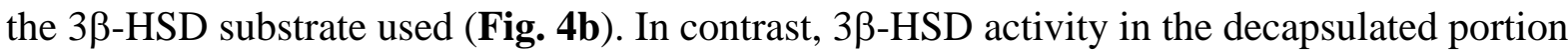
of the adrenal gland (containing the zona fasciculata, zona reticularis and medulla) was essentially unaffected in Cry-null mice (Supplementary Fig. 6). These results indicate that an elevation of 3 $\beta$-HSD activity accompanies the accumulation of Hsd3b6 protein observed in aldosterone-producing zona glomerulosa cells.

We next examined whether pharmacological inhibition of 3 $\beta$-HSD enzymatic activity in vivo by treatment of mice with trilostane ${ }^{33}$ leads to a reduction in the PAC in Cry-null mice. The PAC in WT mice remained nearly unchanged by trilostane treatment even at a relatively 
high standard dose (8 mg per kg body weight per d) (Fig. 4c). The apparent tolerance of WT mice to trilostane treatment has been considered to result from feedback regulation by the RAAS $^{34}$. In marked contrast, trilostane treatment was effective in suppressing the increased PAC in Cry-null mice (Fig. 4c), in which the constitutive overproduction of aldosterone reflects its independence from normal RAAS control. These data are consistent with the idea that the highly elevated $3 \beta$-HSD activity in zona glomerulosa cells of Cry-null mice contributes to the elevated PAC of these mice.

\section{Aldosterone-dependent hypertension in Cry-null mice}

We next explored the pathological consequences of excessive aldosterone in Cry-null mice. As a finely controlled RAAS is crucial to blood pressure homeostasis, we compared blood pressure in WT and Cry-null mice (Fig. 5). We monitored daily changes in the mean arterial blood pressure in constant darkness by radiotelemetry (Supplementary Methods). The circadian variation in blood pressure characteristic for WT mice was completely lost in Cry-null mice (Fig. 5a). However, when averaged over 24 h, blood pressure in WT and Cry-null mice appeared similar under standard conditions (Fig. 5b).

Because mineralocorticoid aldosterone-dependent hypertension may be influenced by dietary salt intake ${ }^{23,24}$, we next examined the salt sensitivity of blood pressure in WT and mutant mice. Normally, blood pressure is resilient to variations in sodium ingestion due to homeostatic control by RAAS. Indeed, blood pressure in WT mice $(n=3)$ was unaffected by the switch from a normal-salt to a high-salt diet (Fig. 5a,b; normal-salt, $100.3 \pm 7.8$ (s.d.) mmHg; high salt, $97.9 \pm 9.4 \mathrm{~mm} \mathrm{Hg}$ ), associated with a marked reduction of PAC upon the high-salt loading (Supplementary Fig. 7). In contrast, Cry-null mice became hypertensive in a salt-dependent manner (Fig. 5a). All mutant mice tested $(n=5)$ experienced a drastic increase in blood pressure within $2 \mathrm{~d}$ after the change to the high-salt diet (Fig. 5b; normal-salt, $97.2 \pm$ 
6.3 (s.d.) mmHg; high-salt, $108.8 \pm 7.9 \mathrm{~mm} \mathrm{Hg} ; P<0.001$, paired $t$ test). The PAC of these mice remained relatively high even after the high-salt loading (Supplementary Fig. 7). The excess of circulating aldosterone indeed contributed to the elevation of blood pressure, as the high-salt-induced hypertension in Cry-null mice $(n=3)$ could be reversed by treatment with eplerenone, a selective aldosterone blocker ${ }^{35}$ (Fig. 5a,b; before eplerenone, $106.7 \pm 5.5$ (s.d.) mmHg; after eplerenone, $99.6 \pm 5.3 \mathrm{~mm} \mathrm{Hg} ; P<0.001$, paired $t$ test). The observed decrease in blood pressure was not due to salt desensitization induced by long-term high salt ingestion, as the blood pressure in Cry-null mice remained elevated while they were fed the high-salt diet (Fig. 5a,b), These data provide evidence for an aldosterone-dependent alteration of blood pressure homeostasis in Cry-null mice.

\section{Human HSD3B1 is a functional counterpart of mouse Hsd3b6}

To begin to address the question of whether these findings have relevance for human idiopathic hypertensive disease $\mathrm{e}^{23,24}$, we tested whether the human adrenal gland also expresses a zona glomerulosa-specific HSD3B isoform. Nontumorous adrenal tissue from adrenalectomy specimens were obtained from two human subjects (2237N and 1774N) after written informed consent. cDNA cloning from zona glomerulosa cells and subsequent DNA sequencing analysis showed that the human HSD3B1 gene, which has been considered a candidate human counterpart of the mouse Hsd3b6 gene $\mathrm{e}^{27,28,36}$ (Supplementary Table 3) was expressed in the human adrenal zona glomerulosa samples tested (Fig. 6a-d).

Strikingly, human HSD3B1 and HSD3B2 show a high sequence similarity (93.6\% identity, including the 5' and 3' untranslated regions; Fig. 6a). We thus developed subtype-specific Taqman minor groove binder (MGB) probes to allow us to distinguish these genes on the basis of nucleotide differences in the region encoding the dehydrogenase catalytic YXXXK motif ${ }^{27}$ (where X is any amino acid): the third residue of the motif encodes histidine in 
HSD3B1 and tyrosine in HSD3B2 (Fig. 6a). Subtype-specific Taqman qRT-PCR analysis revealed that expression of human $H S D 3 B 1$ was considerably enriched in zona glomerulosa cells, as compared to zona fasciculata cells. Conversely, expression of human HSD3B2 was predominantly expressed in zona fasciculata cells, suggesting that this gene is a counterpart of the mouse Hsd3b1 gene (Fig. 6e). These observations support the notion that human HSD3B1, but not HSD3B2, represents a functional counterpart of the mouse Hsd3b6 gene

\section{(Supplementary Table 3).}

\section{DISCUSSION}

The present study unveils a new aspect of circadian clock-related pathology: salt-sensitive hypertension. Cry-null mice show an adrenal disorder characterized by chronic overproduction of aldosterone that persists even in the reduced plasma renin activity. Our search for the pathogenic factor underlying this adrenal disorder led to the identification of Hsd3b6, which to our knowledge has not been previously associated with hypertension in rodents. The circadian clock governs the levels of daily expression of $H s d 3 b 6$. The impediment of the circadian clock could therefore couple to the development of hypertension through the abnormal regulation of Hsd3b6-dependent aldosterone synthesis in the adrenal gland.

How do our findings relate to human aldosterone-dependent hypertensive disease? Chronic overproduction of aldosterone by human adrenal zona glomerulosa cells, a disorder referred to as primary hyperaldosteronism, is clinically known to constitute a frequent form of secondary hypertension $^{23}$. Primary hyperaldosteronism can be classified into two distinct diagnostic categories: aldosterone-producing adenoma and bilateral idiopathic hyperaldosteronism. Unlike aldosterone-producing adenoma, which has a known etiology that can be corrected by surgery, the pathophysiology underlying nontumorigenic idiopathic hyperaldosteronism remains totally unknown ${ }^{24}$, as reflected in its being labeled 'idiopathic'. 
Idiopathic hyperaldosteronism does not allow for surgical correction by adrenalectomy because of its bilateral nature, and specific therapies to reduce aldosterone production are not available ${ }^{23}$. Despite the medical importance of this disorder, the underlying mechanisms have remained unexplored largely because of the lack of a suitable animal model.

Cry-null mice, in which circulating aldosterone is chronically overproduced by the adrenal glands, represent a promising animal model of idiopathic hyperaldosteronism. Our analysis of steroidogenic gene expression in Cry-null zona glomerulosa cells led to an unexpected finding. Whereas the rate-limiting step for aldosterone production by zona glomerulosa cells remains to be experimentally defined, previous studies suggested a key role for Cyp11b2, as the enhancement of aldosterone biosynthesis by angiotensin II is associated with an acute induction of Cyp11b2 expression in zona glomerulosa cells ${ }^{37}$. However, we observed that Cyp11b2 expression was essentially unimpaired in Cry-null mice, suggesting another molecular mechanism for aldosterone overproduction in Cry-null mice. We thus carried out a nonbiased screening, leading us to identify Hsd3b6 as a new gene exclusively expressed in zona glomerulosa cells. Notably, the only effect on gene expression that we were able to detect in Cry-null adrenal glands was increased expression of zona glomerulosa-specific Hsd3b6; the expression of other steroidogenic genes tested was unaffected. Furthermore, elevation of Hsd3b6 protein abundance resulted in chronically enhanced 3 $\beta$-HSD activity in Cry-null adrenal zona glomerulosa cells, and in vivo inhibition of $3 \beta$-HSD activity by trilostane was effective in suppressing the increased PAC in Cry-null mice. These data thus characterize mouse Hsd3b6 as a latent risk factor for hyperaldosteronism: chronic elevation of its activity in zona glomerulosa cells instigates an abnormal elevation of PAC. 
It may be somewhat unexpected that zona glomerulosa-specific HSD3B gene expression by human HSD3B1 and mouse Hsd3b6 has escaped notice thus far. However, it should be noted that to show that human HSD3B1 gene expression is specific to zona glomerulosa cells, we had to develop a Taqman MGB probe-based qPCR method that can distinguish between transcripts of the HSD3B1 and HSD3B2 genes. Notably, these two isoforms are indistinguishable by conventional probe hybridization methods because of their extremely high DNA sequence similarity. Furthermore, as the zona glomerulosa cell population constitutes only a fraction of the total number of adrenal gland cells, the levels of gene expression in this cell type might have been underestimated in assays using whole adrenal extracts $^{38,39}$. Indeed, we observed that the expression of mouse Hsd3b6 was apparently low when analyzed in whole adrenal RNA. By applying laser-microdissection technology, in combination with subtype-specific qRT-PCR, we were able to identify the zona glomerulosa-specific HSD3B genes in both human and mouse adrenal glands.

On the basis of these results, the potential involvement of the human HSD3B1 gene in adrenal zona glomerulosa pathophysiology merits further investigation. Whereas numerous studies link mutations in human HSD3B2 to the 3 $\beta$-HSD deficiency leading to impaired steroidogenesis in both the adrenal glands and the gonads ${ }^{40,41}$, no human HSD3B1 gene mutations have been observed in clinical screening of human postnatal disorders ${ }^{27}$. Human HSD3B1 is expressed not only in adrenal zona glomerulosa but also in the placenta, where HSD3B1 functions as the major 3 $\beta$-HSD isoform for production of progesterone, which is vital for the maintenance of human pregnancy. It was therefore suggested that, in a fetus homozygous for a HSD3B1 gene defect, absence of the placental enzyme would lead to interruption of pregnancy before the end of the first trimester ${ }^{27,42}$. 
Although it is unclear whether decreased expression or function of human HSD3B1 can have pathological effects in the adult, there remains the question of whether its increased expression or function might be associated with adult hyperaldosteronism. Although further clinical investigation will be needed to address this question, the finding of a zona glomerulosa-specific HSD3B gene subtype suggests a potential therapeutic application of subtype-specific HSD3B inhibitors. Historically, individuals with idiopathic hyperaldosteronism have been treated with a broad-spectrum HSD3B inhibitor that has negative side effects owing to its inhibition not only of aldosterone production but also of corticosterone synthesis ${ }^{43,44}$. It is thus tempting to speculate that selective inhibitors for the zona glomerulosa-specific HSD3B subtype may offer new therapeutic treatments for aldosterone control.

In conclusion, we have found an adrenalopathy in circadian clock-deficient Cry-null mice. The inactivation of Cry genes leads to chronically enhanced mineralocorticoid production, which, in turn, renders blood pressure salt-sensitive. Our analysis of the hyperaldosteronemia of these mice led to the discovery of a zona glomerulosa-specific HSD3B subtype. Although it is still not known how overproduced aldosterone causes salt-sensitive hypertension in Cry-null mice, our studies implicating this HSD3B isoform in hypertension may help unravel the etiologies of salt-sensitive hypertension and idiopathic hyperaldosteronism in humans.

\section{ACKNOWLEDGMENTS}

We thank T. Ono, A. Hirasawa, T. Koshimizu, K. Terasawa, H. Nishinaga, M. Sato, Y. Yamaguchi, M. Matsuo, J.M. Fustin, K. Toida, H. Sei and K. Ishimura for technical support and valuable discussion. We also thank T. Michel for critical reading of the manuscript. This work was supported in part by the Specially Promoted Research 
(to H.O.) and Grant-in-Aid for Young Scientists (to M.D.) from the Ministry of Education, Culture, Sports, Science and Technology of Japan and grants from Nakatomi Foundation, SRF (to H.O.), Senri Life Science Foundation, Takeda Science Foundation (to M.D.) and the Netherlands Organization of Scientific research, ZonMW Vici 918.36.619 (to G.T.J.v.d.H.). Trilostane was a generous gift from Mochida Pharmaceutical. Eplerenone was a generous gift from Pfizer.

\section{AUTHOR CONTRIBUTIONS}

M.D. and H.O. designed the research; A.K., O.O., T.T. and G.T.J.v.d.H. supplied the experimental materials; M.D., Y.T., R.K., F.Y., H.Y., S.H., K.T. and H.O. acquired the data; M.D., N.E., Y.O., G.T., K.T. and H.O. analyzed the data; and M.D., G.T.J.v.d.H. and H.O. drafted the manuscript.

1. Staessen, J.A., Wang, J., Bianchi, G. \& Birkenhager, W.H. Essential hypertension. Lancet 361, 1629-1641 (2003).

2. Lifton, R.P., Gharavi, A.G. \& Geller, D.S. Molecular mechanisms of human hypertension. Cell 104, 545-556 (2001).

3. Schibler, U. \& Sassone-Corsi, P. A web of circadian pacemakers. Cell 111, 919-922 (2002).

4. Dunlap, J.C. Molecular bases for circadian clocks. Cell 96, 271-290 (1999). </jrn>

5. Reppert, S.M. \& Weaver, D.R. Coordination of circadian timing in mammals. Nature 418, 935-941 (2002).

6. Wijnen, H. \& Young, M.W. Interplay of circadian clocks and metabolic rhythms. Annu. Rev. Genet. 40, 409-448 (2006). 
7. Takahashi, J.S., Hong, H.K., Ko, C.H. \& McDearmon, E.L. The genetics of mammalian circadian order and disorder: implications for physiology and disease. Nat. Rev. Genet. 9, 764-775 (2008).

8. Panda, S. et al. Coordinated transcription of key pathways in the mouse by the circadian clock. Cell 109, 307-320 (2002).

9. Green, C.B., Takahashi, J.S. \& Bass, J. The meter of metabolism. Cell 134, 728-742 (2008).

10. Hastings, M.H., Reddy, A.B. \& Maywood, E.S. A clockwork web: circadian timing in brain and periphery, in health and disease. Nat. Rev. Neurosci. 4, 649-661 (2003).

11. Furlan, R. et al. Modifications of cardiac autonomic profile associated with a shift schedule of work. Circulation 102, 1912-1916 (2000).

12. Bradley, T.D. \& Floras, J.S. Sleep apnea and heart failure: Part II: central sleep apnea. Circulation 107, 1822-1826 (2003).

13. Scheer, F.A., Hilton, M.F., Mantzoros, C.S. \& Shea, S.A. Adverse metabolic and cardiovascular consequences of circadian misalignment. Proc. Natl. Acad. Sci. USA 106, 4453-4458 (2009).

14. van der Horst, G.T. et al. Mammalian Cry1 and Cry2 are essential for maintenance of circadian rhythms. Nature 398, 627-630 (1999).

15. Vitaterna, M.H. et al. Differential regulation of mammalian period genes and circadian rhythmicity by cryptochromes 1 and 2. Proc. Natl. Acad. Sci. USA 96, 12114-12119 (1999).

16. Matsuo, T. et al. Control mechanism of the circadian clock for timing of cell division in vivo. Science 302, 255-259 (2003). 
17. Yamaguchi, S. et al. Role of DBP in the circadian oscillatory mechanism. Mol. Cell. Biol. 20, 4773-4781 (2000).

18. Kume, K. et al. mCRY1 and mCRY2 are essential components of the negative limb of the circadian clock feedback loop. Cell 98, 193-205 (1999).

19. Okamura, H. et al. Photic induction of mPer1 and mPer2 in cry-deficient mice lacking a biological clock. Science 286, 2531-2534 (1999).

20. Mitsui, S., Yamaguchi, S., Matsuo, T., Ishida, Y. \& Okamura, H. Antagonistic role of E4BP4 and PAR proteins in the circadian oscillatory mechanism. Genes Dev. 15, 995-1006 (2001).

21. Ishida, A. et al. Light activates the adrenal gland: timing of gene expression and glucocorticoid release. Cell Metab. 2, 297-307 (2005).

22. Balsalobre, A. et al. Resetting of circadian time in peripheral tissues by glucocorticoid signaling. Science 289, 2344-2347 (2000).

23. Young, W.F. Primary aldosteronism: renaissance of a syndrome. Clin. Endocrinol. 66, 607-618 (2007).

24. Kaplan, N.M. Primary aldosteronism. in Clinical Hypertension 410-433 (Lippincott Williams \& Wilkins, Philadelphia, 2006).

25. Brown, N.J. Eplerenone: cardiovascular protection. Circulation 107, 2512-2518 (2003).

26. Gomez-Sanchez, C., Holland, O.B., Higgins, J.R., Kem, D.C. \& Kaplan, N.M. Circadian rhythms of serum renin activity and serum corticosterone, prolactin and aldosterone concentrations in the male rat on normal and low-sodium diets. Endocrinology 99, 567-572 (1976). 
27. Simard, J. et al. Molecular biology of the $3 \beta$-hydroxysteroid dehydrogenase $/ \Delta^{5}-\Delta^{4}$ isomerase gene family. Endocr. Rev. 26, 525-582 (2005).

28. Payne, A.H. \& Hales, D.B. Overview of steroidogenic enzymes in the pathway from cholesterol to active steroid hormones. Endocr. Rev. 25, 947-970 (2004).

29. Giroud, C.J., Stachenko, J. \& Venning, E.H. Secretion of aldosterone by the zona glomerulosa of rat adrenal glands incubated in vitro. Proc. Soc. Exp. Biol. Med. 92, 154-158 (1956).

30. Domalik, L.J. et al. Different isozymes of mouse $11 \beta$-hydroxylase produce mineralocorticoids and glucocorticoids. Mol. Endocrinol. 5, 1853-1861 (1991).

31. Ogishima, T., Suzuki, H., Hata, J., Mitani, F. \& Ishimura, Y. Zone-specific expression of aldosterone synthase cytochrome P-450 and cytochrome P-45011 $\beta$ in rat adrenal cortex: histochemical basis for the functional zonation. Endocrinology 130, 2971-2977 (1992).

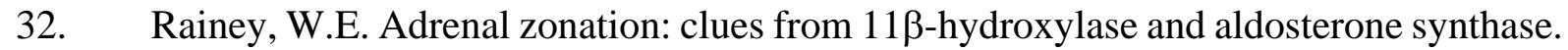
Mol. Cell. Endocrinol. 151, 151-160 (1999).

33. Potts, G.O., Creange, J.E., Hardomg, H.R. \& Schane, H.P. Trilostane, an orally active inhibitor of steroid biosynthesis. Steroids 32, 257-267 (1978).

34. Jungmann, E. et al. The inhibiting effect of trilostane on adrenal steroid synthesis: hormonal and morphological alterations induced by subchronic trilostane treatment in normal rats. Res. Exp. Med. (Berl.) 180, 193-200 (1982).

35. Brown, R., Quirk, J. \& Kirkpatrick, P. Eplerenone. Nat. Rev. Drug Discov. 2, 177-178 (2003). 
36. Mason, J.I. et al. The regulation of 3 $\beta$-hydroxysteroid dehydrogenase expression. Steroids 62, 164-168 (1997).

37. Makhanova, N., Hagaman, J., Kim, H.S. \& Smithies, O. Salt-sensitive blood pressure in mice with increased expression of aldosterone synthase. Hypertension 51, 134-140 (2008).

38. Rhéaume, E. et al. Structure and expression of a new complementary DNA encoding the almost exclusive $3 \beta$-hydroxysteroid dehydrogenase $/ \Delta^{5}-\Delta^{4}$-isomerase in human adrenal glands and gonads. Mol. Endocrinol. 5, 1147-1157 (1991).

39. Abbaszade, I.G. et al. Isolation of a new mouse 3 $\beta$-hydroxysteroid dehydrogenase isoform, 3 $\beta$-HSD VI, expressed during early pregnancy. Endocrinology 138, 1392-1399 (1997).

40. Moisan, A.M. et al. New insight into the molecular basis of 3ß-hydroxysteroid dehydrogenase deficiency: identification of eight mutations in the HSD3B2 gene eleven patients from seven new families and comparison of the functional properties of twenty-five mutant enzymes. J. Clin. Endocrinol. Metab. 84, 4410-4425 (1999).

41. Rhéaume, E. et al. Congenital adrenal hyperplasia due to point mutations in the type II 3ß-hydroxysteroid dehydrogenase gene. Nat. Genet. 1, 239-245 (1992).

42. Peng, L., Arensburg, J., Orly, J. \& Payne, A.H. The murine 3 $\beta$-hydroxysteroid

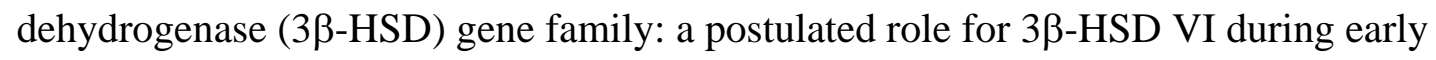
pregnancy. Mol. Cell. Endocrinol. 187, 213-221 (2002).

43. Nakada, T. et al. Primary aldosteronism treated by trilostane (3 $\beta$-hydroxysteroid dehydrogenase inhibitor). Urology 25, 207-214 (1985). 
44. Winterberg, B., Vetter, W., Groth, H., Greminger, P. \& Vetter, H. Primary aldosteronism: treatment with trilostane. Cardiology 72 Suppl 1, 117-121 (1985).

Figure 1 Overproduction of aldosterone by Cry-null adrenal glands. (a) Twenty-four-hour profiles of PAC in WT and Cry-null mice in constant darkness ( $n=5-12$ mice per time point). The values at CT0 are double plotted. (b) Plasma renin activity in WT and Cry-null mice at CT0 and CT12 ( $n=6$ mice per time point). (c) Aldosterone secretion from ex vivo tissue slices of adrenal glands collected from WT and Cry-null mice at CT0 and CT12 (CT0: WT, $n=6$, Cry-null, $n=6$; CT12: WT, $n=12$, Cry-null, $n=10$ ). Shown are the amounts of aldosterone secreted into serum-free medium during a 60 -min incubation at $37^{\circ} \mathrm{C}$. Values in a-c are means \pm s.e.m. $* P<0.05 ; * * P<0.01 ; * * * P<0.001$.

Figure 2 Chronically high expression of $H s d 3 b 6$ in aldosterone-producing zona glomerulosa (ZG) cells of Cry-null adrenal glands. (a) Twenty-four-hour expression profiles of steroidogenic genes in WT and Cry-null adrenal glands. The relative levels of each transcript, determined by DNA microarray analysis, are indicated by the color scale. For each transcript, values were normalized to the average expression level over the day in the WT adrenal gland. The identity of each transcript is declared by Affymetrix probe used, with gene symbol (see also gene prefixes shown in Supplementary Methods) except that three isoforms of $H s d 3 b$ gene (Hsd3b2, Hsd3b3 and Hsd3b6), to which the gene probe 1460232_s_at can hybridize, are denoted as Hsd3b2/3/6. (b) qRT-PCR-verified 24-h expression profiles of Hsd3b6 and Hsd3b1 in WT and Cry-null adrenal glands. Values are means \pm s.e.m. ( $n=5$ mice for each data point) normalized to expression of TATA-box binding protein (Tbp)-encoding gene. The middle graph is an expanded version of the left graph, clarifying the circadian rhythmicity (phase and amplitude) of Hsd3b6 in WT adrenal gland. $* P<0.05$; ** $P<0.01$. (c) Radioisotopic in situ 
hybridization autoradiographs for Hsd3b6, Hsd3b1, Cyp11b2 and Cyp11b1 in WT and Cry-null adrenal glands collected at CT0. Scale bars, $0.5 \mathrm{~mm}$. (d) Zonal expression of Hsd3b and Cyp $11 b$ isoforms. cDNA fragments of $H s d 3 b$ and $C y p 11 b$ were amplified from either ZG or zona fasciculata (ZF) cells as indicated and were enzymatically digested with isoform-specific restriction endonucleases. I, digestion with AccI; VI, NdeI; CI, NheI; CII, SacI. U, undigested; M, DNA marker. The micrographs show a representative tissue section before and after laser microdissection. ZG cells are ovoid in shape and are arranged in globular clusters, lying directly beneath the capsular layer. C, capsule; ZR, zona reticularis. Toluidine blue staining. Scale bars, $50 \mu \mathrm{m}$. The data shown in (c) and (d) are representative of three or more independent experiments. (e) Left, schematic of the aldosterone biosynthetic pathway. Right, qRT-PCR analysis of the expression of genes involved in aldosterone biosynthesis in WT and Cry-null ZG cells at CT0 and CT12 ( $=4$ adrenals for each cDNA preparation). Values are expressed as the mean of two independent experiments, which yielded equivalent results. Rplp0 is a nonoscillating abundant gene, the expression of which reflects the total amount of RNA used.

Figure 3 Coexpression of Hsd3b6 protein and Cyp11b2 mRNA in adrenal ZG cells. (a) Immunocytochemistry of Hsd3b6 protein in a Cry-null adrenal gland. Scale bar, $100 \mu \mathrm{m}$. (b) Double labeling of Hsd3b6 protein and Cyp11b2 transcript. Immunostained adrenal sections were subsequently subjected to in situ hybridization with a digoxigenin-labeled Cyp11b2 probe. Shown are higher magnification views of a region containing ZG cells. Nearly all ZG cells express both Hsd3b6 (red) and Cyp11b2 (blue). Arrows indicate representative double-labeled cells. Scale bar, $100 \mu \mathrm{m}$. The data are representative of three or more independent experiments.

Figure 4 Elevated 3 $\beta$-HSD activity is responsible for aldosterone overproduction in Cry-null mice. (a) Immunoblot analysis of Hsd3b6 and Cyp11a1 proteins in microsomal protein extracts 
from WT and Cry-null adrenal glands isolated at CT0 and CT12 (n = 4 adrenals for each time point). Similar data were acquired in two independent expreriments. (b) 3 $\beta$-HSD enzymatic activity in the ZG cell layer-containing capsular portion of WT and Cry-null adrenal glands at CT0 and CT12. Left, progesterone synthesis from ${ }^{3} \mathrm{H}$-pregnenolone; right, androstenedione synthesis from ${ }^{3} \mathrm{H}$-dehydroepiandrosterone. We used one adrenal gland per assay. Values are means \pm s.e.m. ( $n=12$ assays for each data point). ${ }^{* *} P<0.0001$. (c) PACs in WT and Cry-null

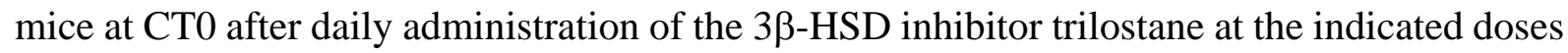
for $7 \mathrm{~d}$ ( $n=6$ mice for each dose). Values are means \pm s.e.m. $\uparrow P=0.01 ; \dagger \dagger P=0.02$.

Figure 5 Cry-null mice show salt-sensitive hypertension. (a) Representative recordings of 15-d temporal profiles of mean arterial blood pressure of WT and Cry-null mice. Mice were kept in constant darkness and fed either normal salt diet (NS; $0.2 \%$ sodium) or high-salt diet (HS; 3.15\% sodium) on the days indicated by gray and yellow arrows, respectively. Blue arrows indicate the period during which the HS diet contained eplenerone (Epl). Gray and black boxes below the graphs represent the subjective day and subjective night, respectively. Blood pressure data were collected in 30-s bouts at 5-min intervals and plotted using a 1-h moving average. The 24-h means of each day are shown by open squares. Horizontal dotted lines indicate the mean value of the blood pressure at days 1-4 on NS diet. (b) Pair-wise comparisons of blood pressures before and after HS diet (left), before and after Epl treatment (middle) and with or without Epl on HS diet (right). Plotted are the blood pressures of individual mice (WT, $n=3$; Cry-null, $n=5$; two of the Cry-null mice were used as non-Epl controls). Values are means \pm s.e.m. ${ }^{* * *} P<0.001$, paired comparison in Cry-null mice.

Figure 6 Identification of a ZG-specific HSD3B isoform in the human adrenal gland. (a) cDNA sequences of human HSD3B1 and HSD3B2. Nucleotides in the protein coding sequence and the 5'- or 3'-untranslated regions are presented by upper and lower case, respectively. 
Dotted line represents HSD3B2 nucleotides that are common to HSD3B1. Asterisks indicate missing nucleotides. Shown in bold are the codons for Y and $\mathrm{K}$ in dehydrogenase catalytic YXXXK motif (X, any amino acid) with the third residue subtype-specific (H for HSD3B1 and Y for HSD3B2). Numbers refer to the nucleotide positions of $H S D 3 B 1$, where +1 corresponds to the initiation of coding sequence. Blue and orange lines above the sequences indicate the probe regions for $H S D 3 B 1$ and $H S D 3 B 2$, respectively. The F0 forward primer (green arrow) recognizes both isoforms, whereas R1 (blue arrow) and R2 (orange arrow) reverse primers are specific to HSD3B1 and HSD3B2, respectively (b) Real-time PCR fluorescence curves for quantification of HSD3B1 mRNA. ZG cells collected from human adrenal samples 2237N and 1774N were subjected to qRT-PCR with F0 and R1 primers. cDNA amplification was readily observed for HSD3B1 (FAM-labeled probe), but not for HSD3B2 (VIC-labeled), showing the specificity of the Taqman probes. $\Delta \mathrm{Rn}$, normalized reporter signal. Similar results were obtained in three independent measurements (c,d) Analysis of cDNA fragments amplified with the F0 and R1 primers. (c) qRT-PCR products were separated by agarose gel electrophoresis. As a control, reverse transcriptase treatment was omitted in lanes 2 and 4 . The arrowhead indicates the expected position (127 base pairs (bp)) of amplicons originating from HSD3B1 transcripts. (d) A representative DNA sequence chromatograph of amplified cDNA fragments from 2237N sample, showing sequence corresponding to the catalytic motif that is unique to HSD3B1. (e) qRT-PCR analysis of HSD3B1 and HSD3B2. The micrograph shows a representative laser microdissection section of the adrenal gland from subject $2237 \mathrm{~N}$. Scale bars, $100 \mu \mathrm{m}$. C, capsule; ZR, zona reticularis; M, medulla. Shown are mean concentrations \pm s.d. (three independent measurements) of HSD3B1 (left) and HSD3B2 (right) in subjects 2237N and1774N. Values were normalized to the expression of RPLP0 and plotted as relative fold of gene expression in the zona fasciculata cells of subject $2237 \mathrm{~N}$. 


\section{ONLINE METHODS}

Mouse experiments. The generation and breeding of Cry-null mice has been described previously ${ }^{14,16,45}$. Before experiments, we housed both WT and Cry-null mice for at least 2 weeks in a 12-h light-dark cycle to synchronize (entrain) the circadian clock of WT mice to the ambient light-dark cycle. We conducted all mouse experiments in constant darkness to eliminate a potential influence of external light on steroidogenesis ${ }^{21}$ and to unequivocally define the effects of the internal biological clock. To eliminate gender- and age-related variations, we routinely used 12 - to 16 -week-old male mice. We transferred the mice into constant darkness, and at selected time points on the first day in constant darkness, we killed the

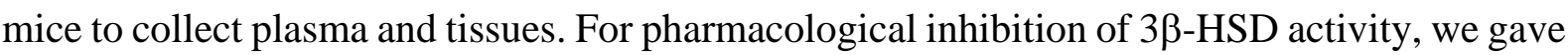
the mice a daily dose of 1.6 or $8.0 \mathrm{mg}$ per kg body weight trilostane (a generous gift from Mochida Pharmaceutical), administered at ZT 6-7 in light-dark cycle (6-7 hr post light onset) by gavage as a suspension in $1 \%$ gum tragacanth. Seven days after the start of medication, we transferred the mice into constant darkness and collected plasma at CT0 in the dark. For salt-loading tests, we fed mice with one of the following diets (CREA Japan Inc.): a normal-salt diet containing $0.2 \%$ elemental $\mathrm{Na}^{+}$, a high-salt diet containing $3.15 \%$ elemental $\mathrm{Na}^{+}$and a high-salt diet with the addition of eplerenone (a generous gift from Pfizer) at a concentration of $1.0 \mathrm{mg}$ per g body weight in chow (estimated intake, $100 \mathrm{mg}$ per kg body weight per d). All of the studies were approved by the Animal Experimentation Committee of Kyoto University.

Adrenal slice culture. Immediately after surgical isolation, we freed adrenal glands (one per assay) of adherent fat and cut them into four slices (about $0.4 \mathrm{~mm}$ thick) with fine scissors. We placed these four fresh slices in $1 \mathrm{ml}$ of serum-free DMEM/F12 medium (Invitrogen), aerated with $5 \% \mathrm{CO}_{2}, 95 \% \mathrm{O}_{2}$ at $37^{\circ} \mathrm{C}$. To wash out the serum carryover in the tissue slices, we preincubated them for 60 min at $37^{\circ} \mathrm{C}$ under a $5 \% \mathrm{CO}_{2}, 95 \% \mathrm{O}_{2}$ gas mixture shaking continuously at a rate of 150 cycles per minute. We replaced the preincubation medium with 1 
$\mathrm{ml}$ of fresh serum-free medium and incubated the slices for 60 min under the same culturing conditions. We determined the amounts of aldosterone secreted into the medium by radioimmunoassay (Coat-A-Count Aldosteone kit, Siemens International).

\section{Biochemical analysis of $3 \beta$-hydroxysteroid dehydrogenase-isomerase activity. We}

mechanically separated the adrenal glands into capsular and decapsulated portions according to the conventional method ${ }^{29}$. We determined 3 $\beta$-HSD enzymatic activities in each portion as previously described ${ }^{46}$. We measured the conversion of $\left[7-{ }^{3} \mathrm{H}\right]$-pregnenolone (PerkinElmer) to ${ }^{3} \mathrm{H}$-progesterone and the conversion of $\left[1,2,6,7-{ }^{3} \mathrm{H}\right]$-dehydroepiandrosterone (PerkinElmer) to ${ }^{3} \mathrm{H}$-androstenedione. Briefly, we incubated cell extracts containing $5 \mu \mathrm{g}$ of protein in reaction buffer (10 mM phosphate, pH 7.5, $140 \mathrm{mM} \mathrm{NaCl,} \mathrm{4 \%} \mathrm{propylene} \mathrm{glycol)} \mathrm{containing} \mathrm{either} 100$ $\mathrm{nM}\left[{ }^{3} \mathrm{H}\right]$-pregnenolone plus $10 \mu \mathrm{M}$ pregnenolone or $100 \mathrm{nM}\left[{ }^{3} \mathrm{H}\right]$-dehydroepiandrosterone plus $15 \mu \mathrm{M}$ dehydroepiandrosterone for $5 \mathrm{~min}$ at $37^{\circ} \mathrm{C}$. After incubation, we extracted steroids by ethyl acetate and subjected them to HPLC analysis by using a reversed-phase column, LiChrospher $100 \mathrm{RP}-18$ (4.0 mm × $250 \mathrm{~mm}$; Kanto). We eluted the column with a 30-min linear gradient of $40-70 \%$ acetonitrile at a flow rate of $0.7 \mathrm{ml} \mathrm{min}^{-1}$, followed by an isocratic elution of $70 \%$ acetonitrile. We fractionated the eluate every minute from 5 to 35 min and counted radioactivity in a liquid scintillation counter.

\section{Taqman quantitative reverse-transcription PCR analysis of human HSD3B1 and}

HSD3B2. We obtained human adrenalectomy specimens from Kyoto University Hospital with written informed consent. All human studies were approved by the Ethics Committee of Kyoto University Graduate School of Medicine. We collected cells in the zona glomerulosa or zona fasciculata separately by laser microdissection (Supplementary Methods). The high sequence similarity between human HSD3B1 and HSD3B2 (Fig. 6a) and the resultant potential cross-hybridization of their probes ${ }^{38}$ were surmounted using Taqman MGB-containing 
fluorogenic probes, which can discriminate accurately between DNA fragments with only a few base pair mismatches ${ }^{47}$. We used the following Taqman probes: for HSD3B1 (NM_000862.2), 5'-FAM-CCATACCCACACAGC-NFQ-MGB-3' (NFQ: non fluorescent quencher) and for HSD3B2 (NM_000198), 5'-VIC-TCCATACCCGTACAGCA-NFQ-MGB-3'. We amplified HSD3B1 and HSD3B2 cDNA fragments with F0 and R1 primers and with F0 and R2 primers, respectively (F0: 5'-AGAAGAGCCTCTGGAAAACACATG-3', R1:

5'-TAAGGCACAAGTGTACAGGGTGC-3'; R2:

5'-CGCACAAGTGTACAAGGTATCACCA-3'). We added the Taqman probes for HSD3B1 and HSD3B2 together (250 $\mathrm{nM}$ each) to the reaction mixtures, as the use of different reporter dyes for each probe with separated emission wavelength maxima (probes for HSD3B1 and HSD3B2 are labeled with FAM and VIC, respectively) enabled simultaneous monitoring of HSD3B1- and HSD3B2-specific amplification in a single PCR tube. We performed real-time PCR with the StepOnePlus instrument (Applied Biosystems). We analyzed cDNA amplification curves with Sequence Detector software (Applied Biosystems), and we expressed the fluorescence of hybridized probes as $\Delta \mathrm{Rn}$ (normalized reporter signal). We normalized the expression of HSD3B1 and HSD3B2 to human RPLP0 (NM_053275), which we evaluated by SYBR Green qPCR analysis (Supplementary Methods).

Statistical analyses. We analyzed the blood pressure changes of individual mice with the two-tailed paired Student's $t$ test. For any other experiments in which three or more test groups were compared, we used one-way analysis of variance with Tukey's post hoc tests and evaluated the differences derived from genotypes, drug application and circadian time points tested. 
Additional methods. Specific reagents and detailed methodology for the determination of PAC and PRA, DNA microarray analysis, isoform specification of $H s d 3 b$ and Cyp $11 b$, promoter analysis of $H s d 3 b 6$, laser microdissection analysis of adrenal sections, in situ hybridization, immunohistochemistry, immunoblotting of adrenal microsomal extracts, qRT-PCR analysis of steroidogenic genes and radiotelemetric monitoring of blood pressure are all described in the Supplementary Methods.

45. Maeda, A. et al. Circadian intraocular pressure rhythm is generated by clock genes. Invest. Ophthalmol. Vis. Sci. 47, 4050-4052 (2006).

46. Matsunaga, M., Ukena, K., Baulieu, E.E. \& Tsutsui, K. 7 $\alpha$-hydroxypregnenolone acts as a neuronal activator to stimulate locomotor activity of breeding newts by means of the dopaminergic system. Proc. Natl. Acad. Sci. USA 101, 17282-17287 (2004).

47. Kutyavin, I.V. et al. 3'-minor groove binder-DNA probes increase sequence specificity at PCR extension temperatures. Nucleic Acids Res. 28, 655-661 (2000). 

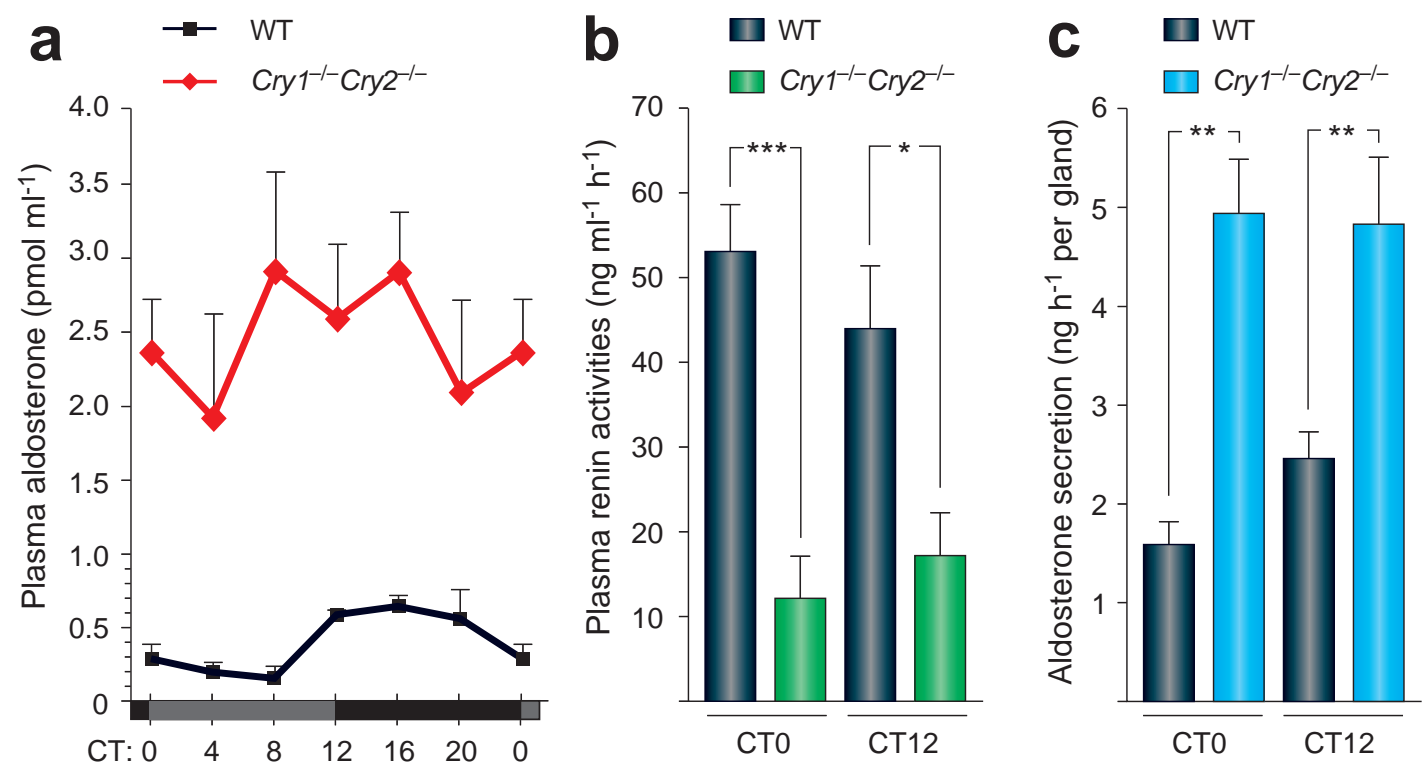

Figure 1 


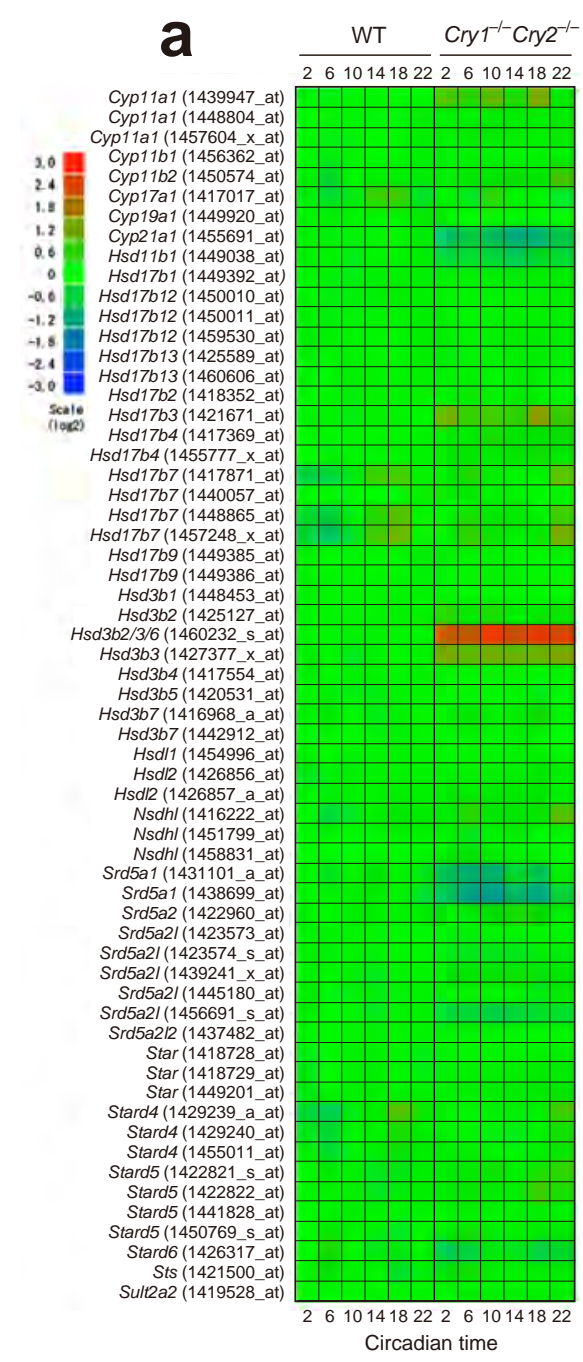

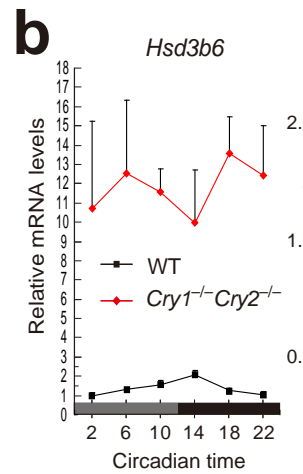

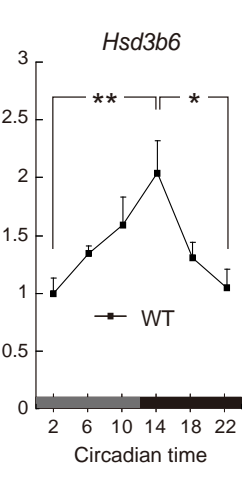

\section{d}
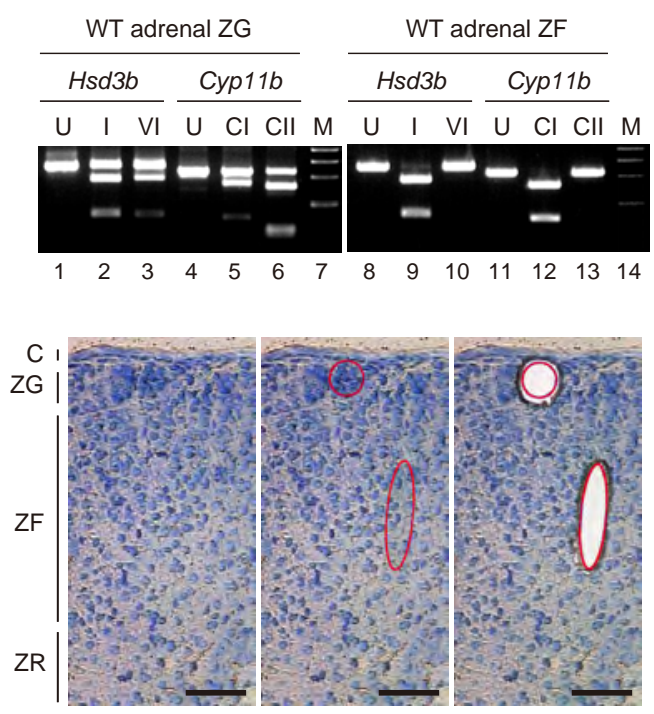
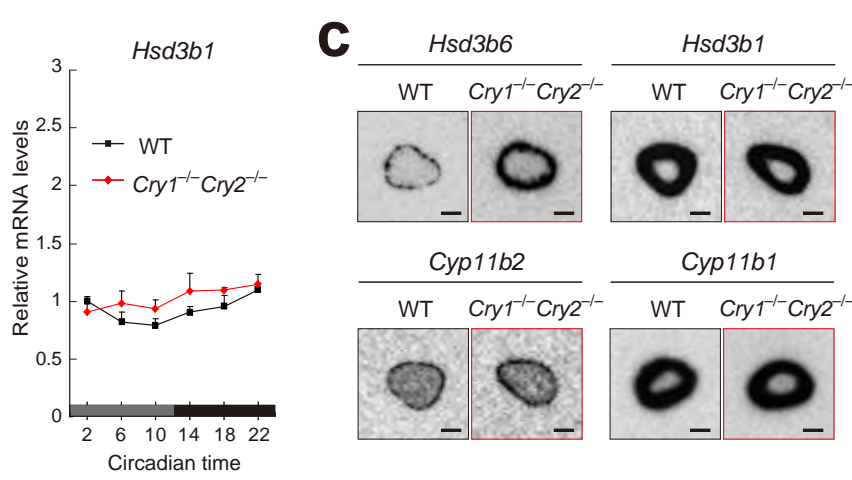

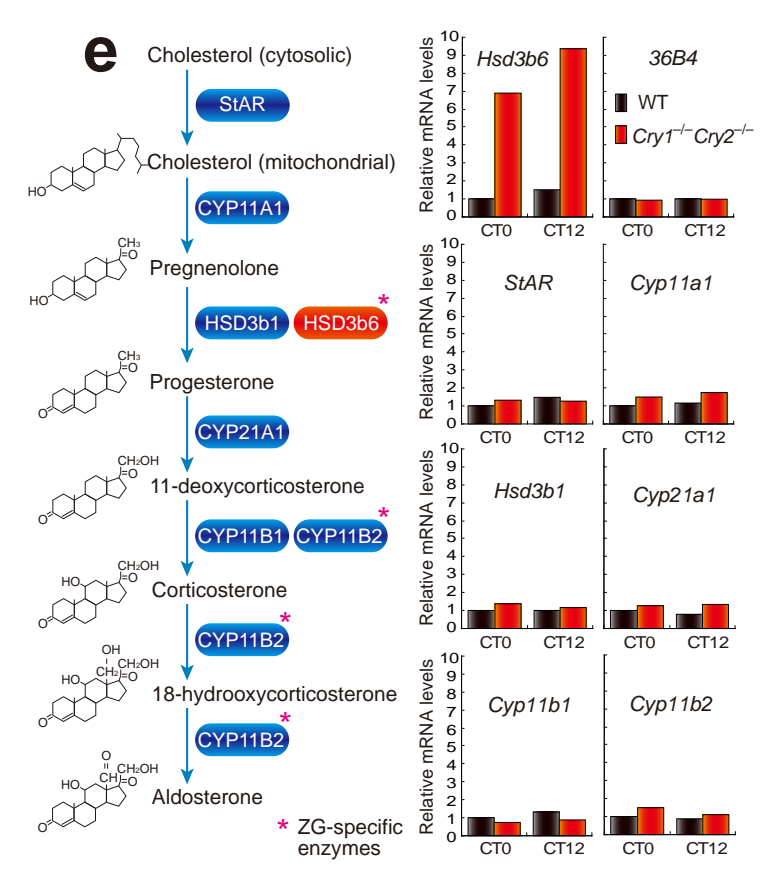

Figure 2 


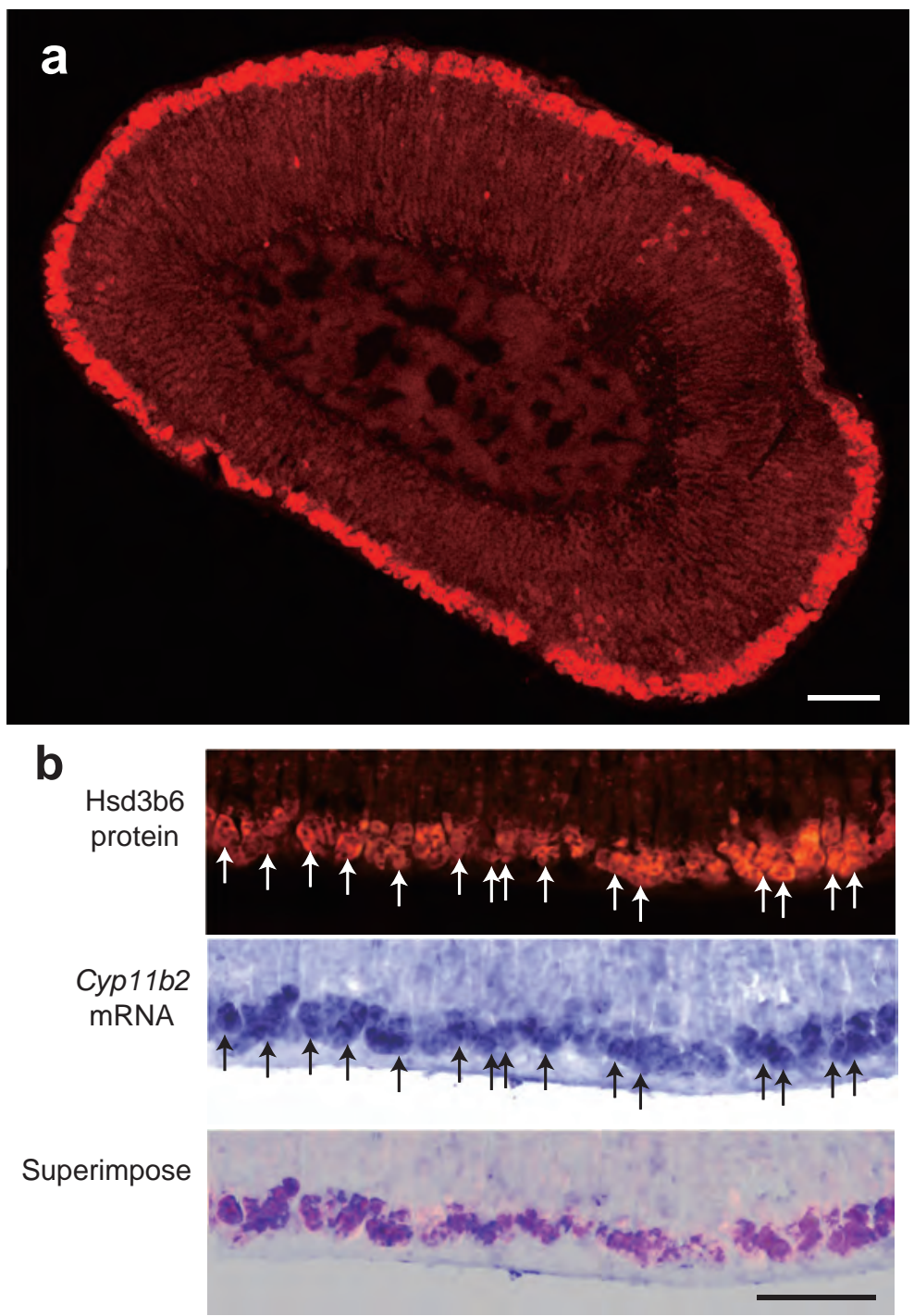

Figure 3 

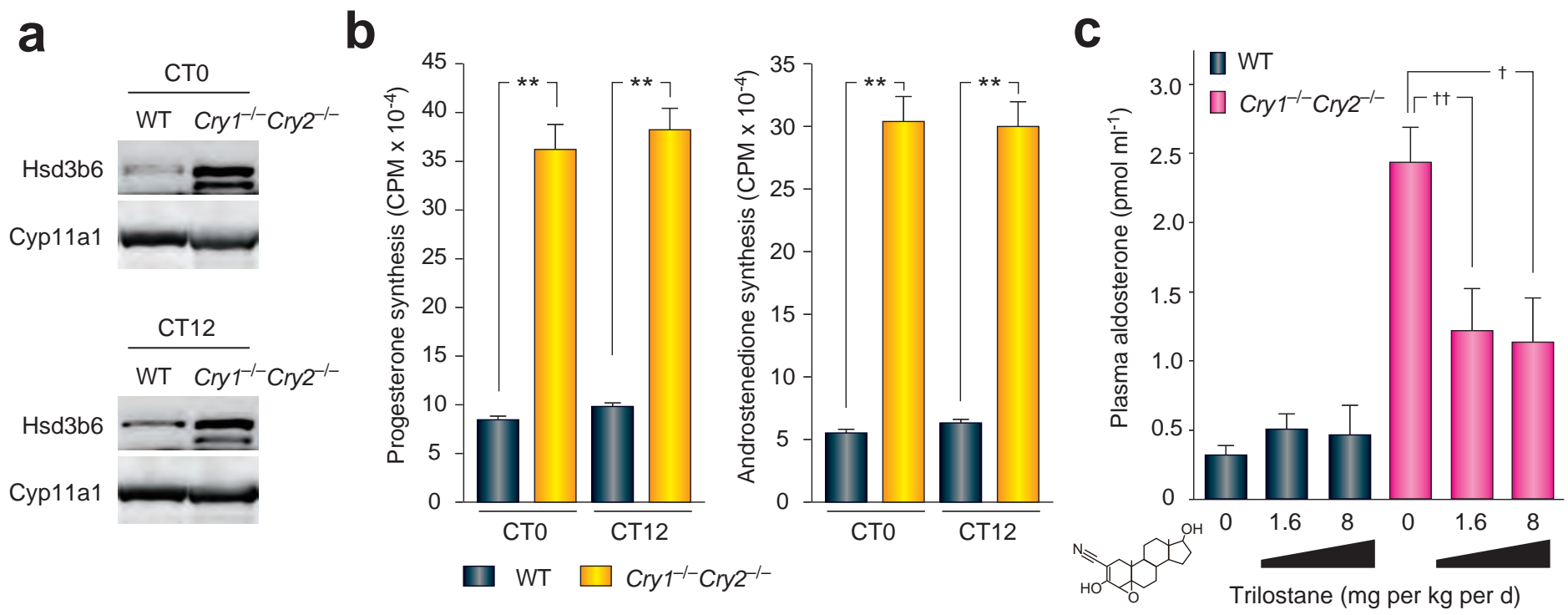

Figure 4 


\section{a}
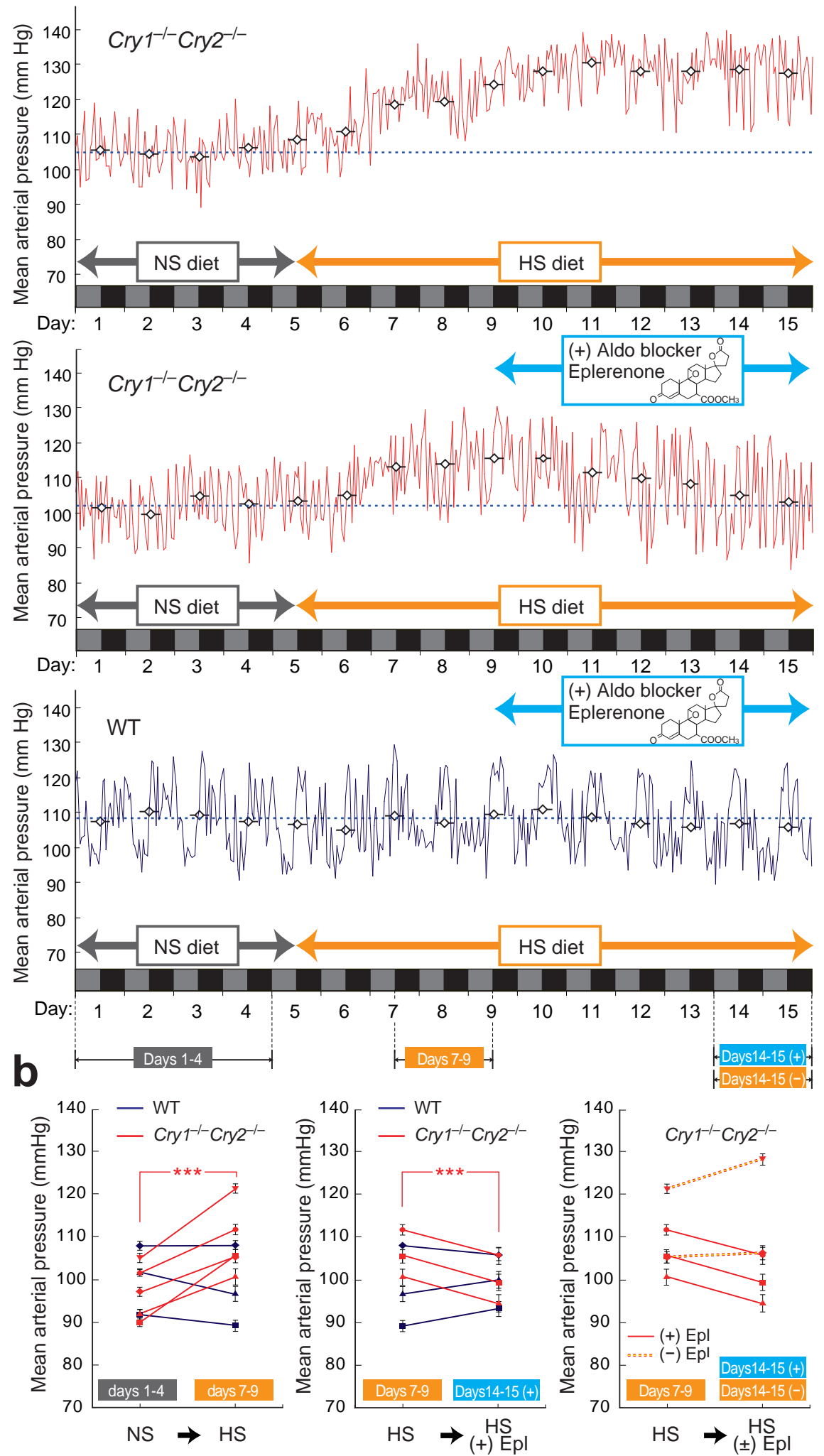

Figure 5 


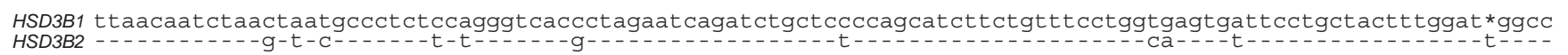

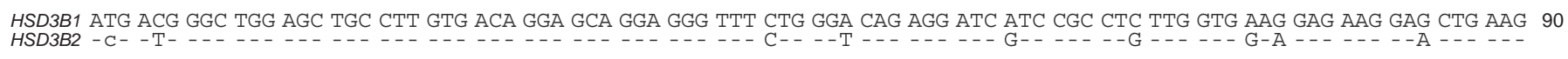

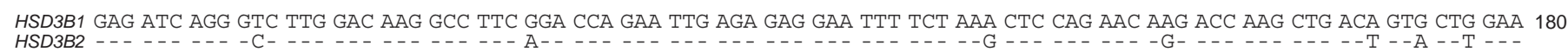
HSD3B1 GGA GAC ATT CTG GAT GAG CCA TTC CTG AAG AGA GCC TGC CAG GAC GTC TCG GTC ATC ATC CAC ACC GCC TGT ATC ATT GAT GTC TTC GGT 270 HSD3B1 GTC ACT CAC AGA GAG TCT ATC ATG AAT GTC AAT GTG AAA GGT ACC CAG CTC CTC TTA GAC GCC TGT GTC CAA GCT AGT GTG CCA GTC TTC 360

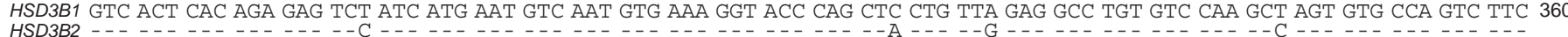
FO primer HSD3B1 ATC TAC ACC AGT AGC ATA GAG GTA GCC GGG CCC AAC TCC TAC AAG GAA ATC ATC CAG AAT GGC CAT GAA GAA GAG CCT CTG GAA AAC ACA 450 HSD3B2 - .

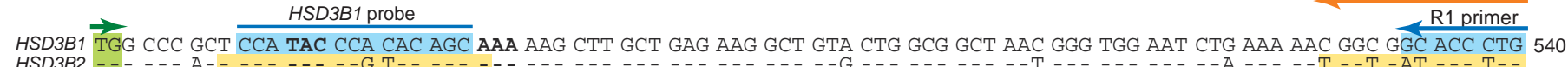

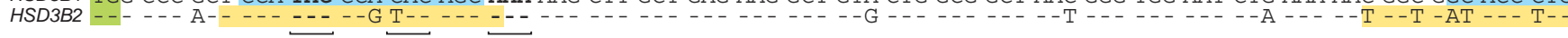
$\frac{Y}{\mathrm{H} / \mathrm{Y}} \quad \frac{-\cdots}{\mathrm{K}}$

HSD3B1
TAC ACT TGT GCC TTA CGA CCC ATG TAT ATC TAT GGG GAA GGA AGC CGA TTC CTT TCT GCT AGT ATA AAC GAG GCC CTG AAC AAC AAT GGG 630

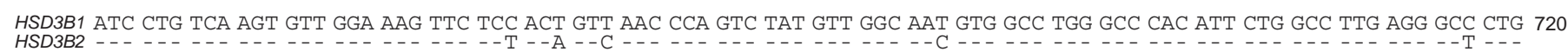
HSD3B2 HSD3B1 CAG GAC CCC AAG AAg GCC CCA AGC ATC CGA GGA CAG TTC TAC TAT ATC TCA GAT GAC ACG CCT CAC CAA AGC TAT GAT AAC CTT AAT TAC 810
HSD3B2 - G-

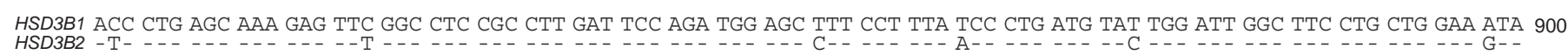
HSD3B2 - T

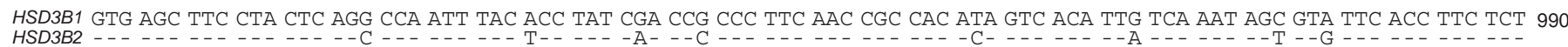
HSD3B1 TAT AAG AAG GCT CAG CGA GAT CTG GCG TAT AAG CCA CTC TAC AGC TGG GAG GAA GCC AAG CAG AAA ACG GTG GAG TGG GTT GGT TCC CTT 1080

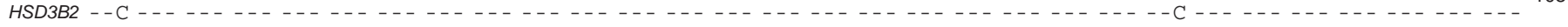
HSD3B1 GTG GAC CGG CAC AAG GAG ACC CTG AAG TCC AAG ACT CAG TGA tttaaggatgacagagatgtgcatgtgggtattgttaggagatgtcatcaagctC 1177

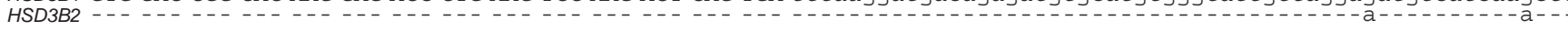
HSD3B1 caccCtcctggcctcatacagaaagtgacaagggcacaagctcaggtcctgctgcctccctttcatacaatggccaacttattgtattcctcatgtcatcaaaa 1281 (1)

HSD3B1 cCtgcgcagtcattggcccaacaagaaggtttctgtcctaatcatataccagaggaaagaccatgtggtttgctgttaccaaatctcagtagctgattctgaac 1385

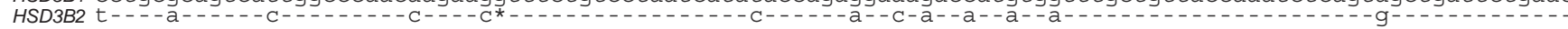

HSD3B1 aatttagggactctttaacttgagggtcg*ttttgactactagagctccatttctactcttaaatgagaaaggatttcctttctttttaatcttccattcctt 1488

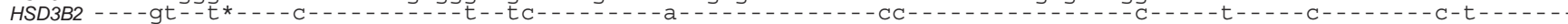

HSD3B1 cacatagtttgataaaaagatcaataaatgtttgaatgtttaatgtgaaaaaaaaa 1544

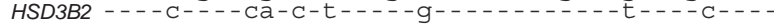

b

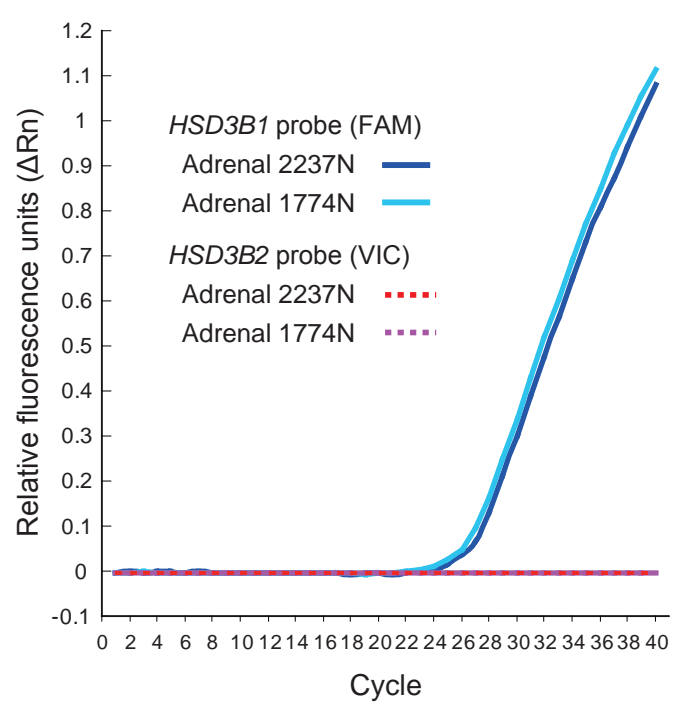

d

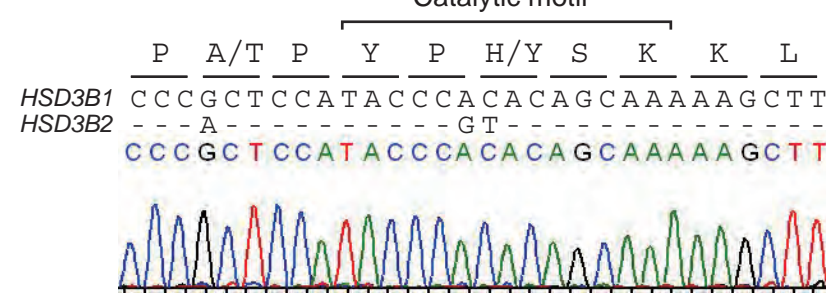

C

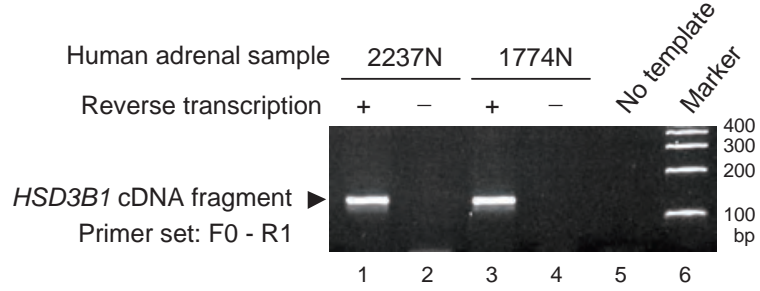

e
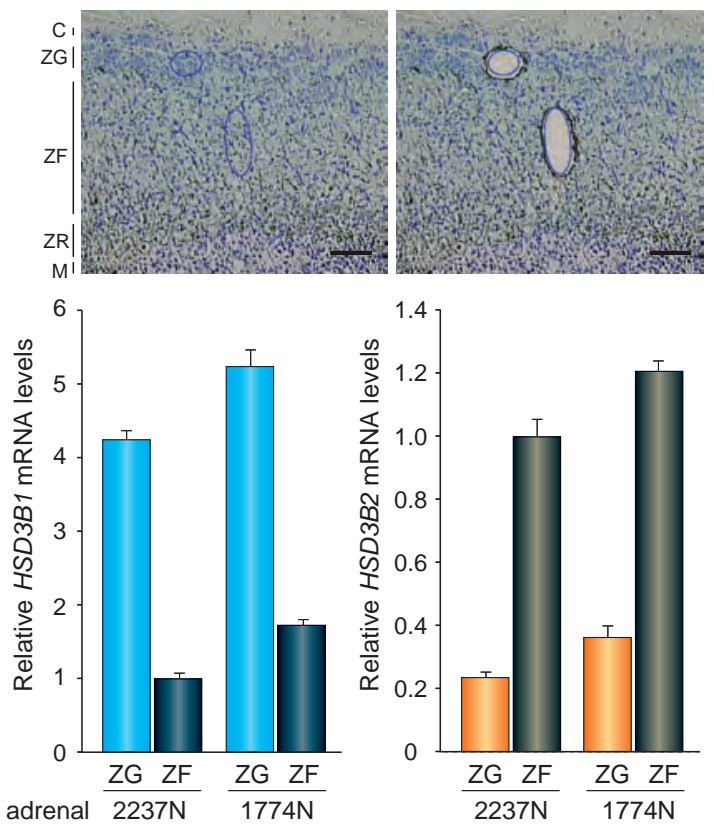


\title{
Elevated adrenal Hsd3b6 underlies salt-sensitive hypertension in circadian clock-deficient Cry mice
}

\author{
Masao Doi, Yukari Takahashi, Rie Komatsu, Fumiyoshi Yamazaki, Hiroyuki Yamada, Shogo \\ Haraguchi, Noriaki Emoto, Yasushi Okuno, Gozoh Tsujimoto, Akihiro Kanematsu, Osamu \\ Ogawa, Takeshi Todo, Kazuyoshi Tsutsui, Gijsbertus T.J. van der Horst \& Hitoshi Okamura
}
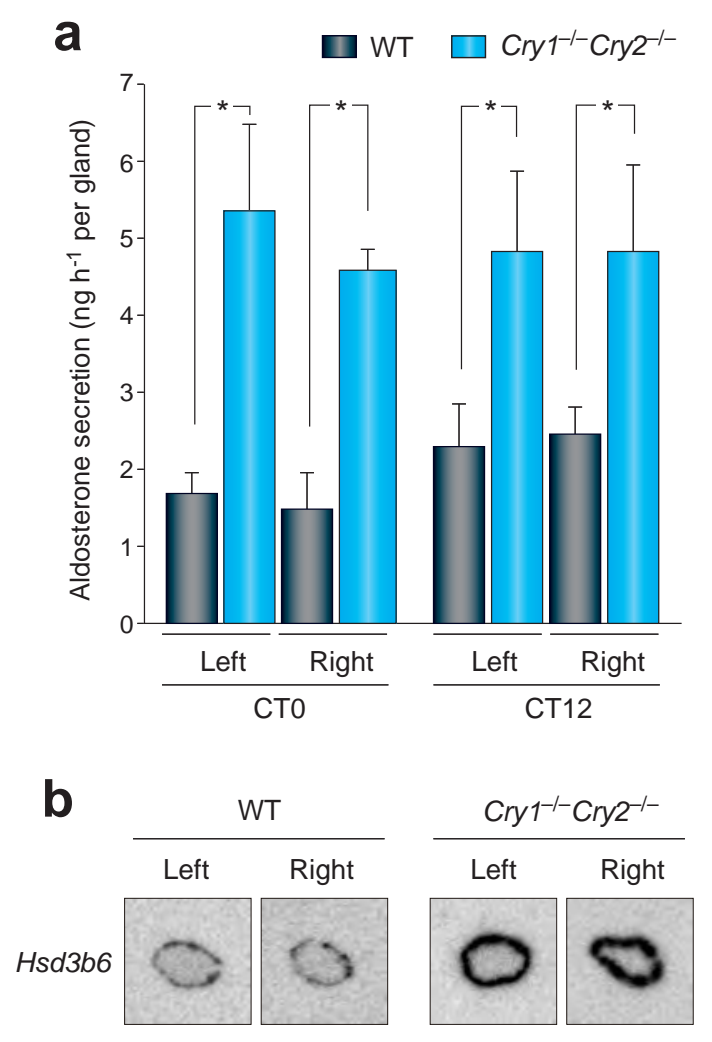

Supplementary Figure 1 Disorders of Cry-null adrenal glands are bilateral. (a) Bilateral over-production of aldosterone in Cry-null adrenals. The amount of aldosterone, released from tissue culture slices of either left or right adrenal glands were measured as described in Fig. 1c. Plotted are the amounts of aldosterone secreted from WT and Cry-null adrenals at CT0 and CT12. Values are mean \pm s.e.m (WT adrenals: CT0, left and right, $n=3$ each; CT12, left and right, $n=6$ each; Cry-null adrenals: CT0, left and right, $n=3$ each; CT12, left and right, $n=5$ each). ${ }^{*} P<0.05$. (b) Bilateral hyperexpression of $H s d 3 b 6$ in Cry-null adrenals. Expression of Hsd3b6 in either left or right adrenal glands was analyzed by in situ hybridization. Shown are representative micrographs of WT and Cry-null adrenals, collected at CT0. 


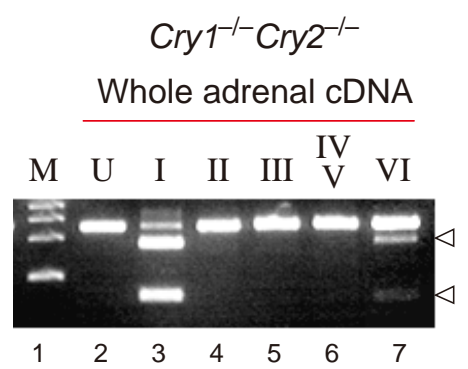

Supplementary Figure 2 Specification of the Hsd3b subtypes expressed in the Cry-null adrenal gland. Whole adrenal total RNA extract of Cry-null mice was analyzed by RT-PCR using a primer set that can amplify all classes of Hsd3b (sequence similarity enabled the amplification with a single PCR with a common primer set). Aliquots of the PCR products were incubated with isoform specific restriction endonucleases (as determined from the DNA sequence) to diagnose the identity of the PCR products: I, AccI; II, HindIII; III, BstBI; IV and V, AvaI (AvaI digests both IV and V); VI, NdeI. U, undigested, M, DNA marker. Arrowheads indicate the digested Hsd3b6 fragments separated by agarose gel electrophoresis. Note that Hsd3b6 is expressed exclusively in the aldosterone-producing ZG cells (see in situ hybridization of Hsd3b6 in Fig. 2c and Supplementary Fig. 5). Since the ZG cell population only constitutes a fraction of the total number of adrenal gland cells, the concentration of Hsd3b6 transcripts in whole adrenal pool (lane 7) was apparently lower than that of Hsd3b1 (lane 3), which is widely expressed in the adrenal cortex (see Fig. 2c). The types II to $\mathrm{V}$ (lanes 4-6) were virtually undetectable. 
a
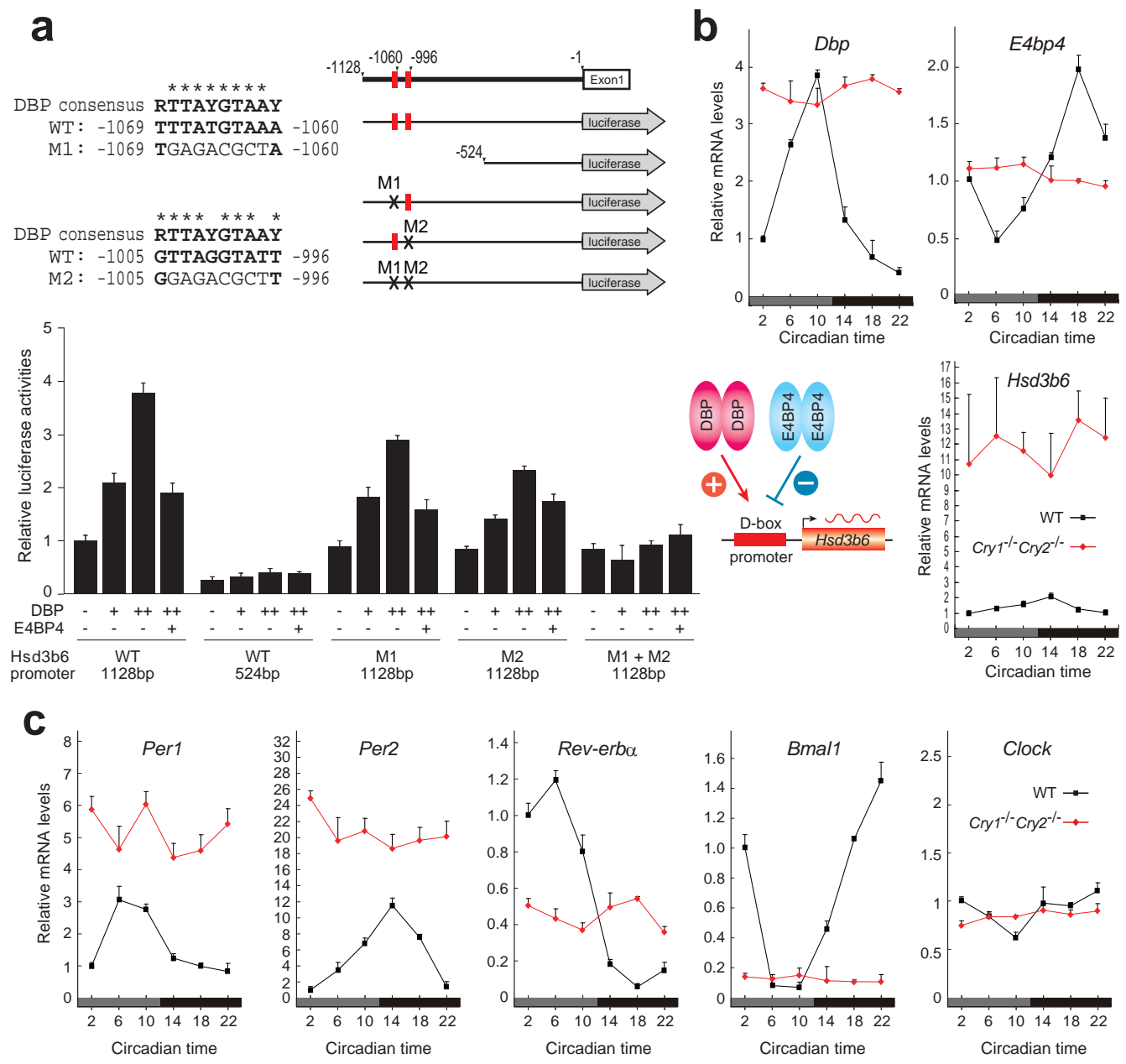

d

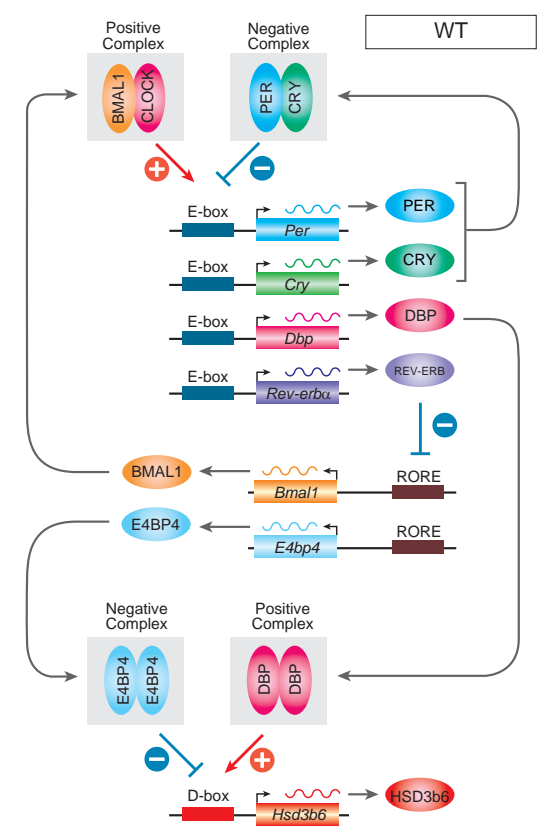

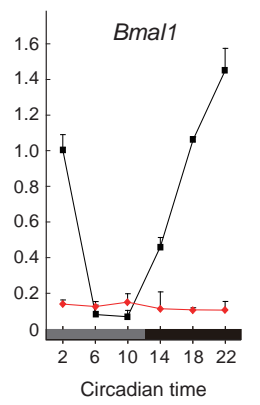
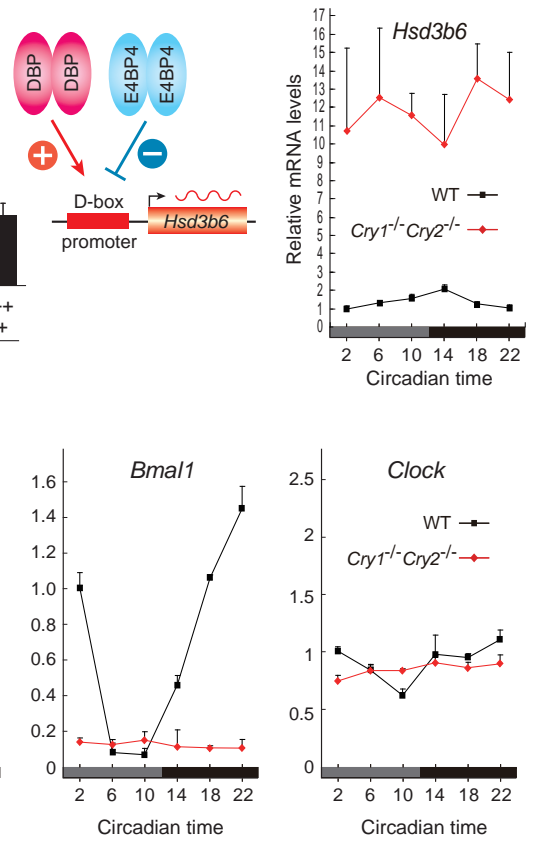

Circadian time

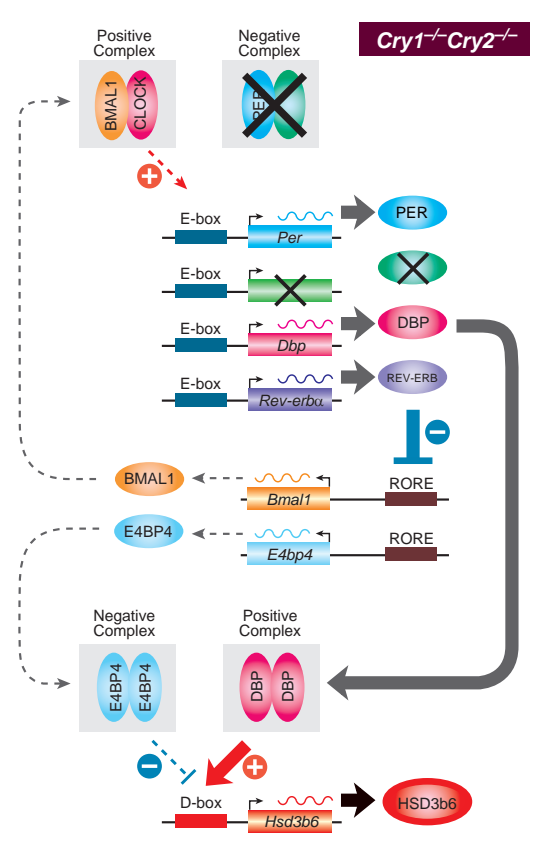

Supplementary Figure 3 Transcriptional control of Hsd3b6 by the circadian regulators DBP and E4BP4. (a) Promoter analysis of the mouse Hsd3b6 gene. The $H s d 3 b 6$ promoter lacks an E-box, but instead contains two functional D-box elements, allowing (i) DBP to activate transcription and (ii) E4BP4 to antagonize DBP-mediated transcription. Transcription assays were performed using H295R cells transiently expressing the luciferase reporter constructs from (mutagenized) promoter regions (as indicated). Error bars indicate the s.e.m from three replicate experiments. (b) Constitutive activation of $D b p$ in the Cry-null adrenal gland. The circadian expression of Dbp and E4bp4, exhibiting anti-phase rhythms in the WT adrenal gland (black lines), was completely abolished in the Cry-null adrenal gland (red lines). Intriguingly, the expression of Dbp was highly elevated throughout the day in the Cry-null adrenal gland, while E4bp4 was expressed at intermediate levels. Error bars indicate the s.e.m $(n=4$, each), normalized to expression of Tbp. (c) Abnormal expression of circadian clock genes in the Cry-null adrenal gland. Similar to $D b p$, the expression of the E-box-regulated Per genes was highly elevated in the Cry-null adrenal gland. Relative mRNA levels were determined by qRT-PCR. Error bars indicate the s.e.m ( $n=4$, each), normalized to expression of Tbp. (d) Schematic model of abnormal control of Hsd3b6 by the circadian regulators DBP and E4BP4. In the $\mathrm{WT}$ adrenal gland, transcription of Hsd3b6 is under the well-balanced circadian control by DBP and E4BP4. In the Cry-null adrenal gland, however, constitutive activation of DBP disrupts the equilibrium, leading to imbalanced hyperexpression of Hsd3b6.

The molecular mechanism underlying the abnormal regulation of Hsd3b6 in the ZG cell (Fig. 2) is also worthy of investigation to understand the etiology behind the adrenal-autonomous circadian disorder (Fig. 1). We observed that the gene promoter of Hsd3b6 is controlled by the circadian regulators DBP (ref. 18) and E4BP4 (refs. 20,48). Specifically, the Hsd3b6 promoter contains two functional D-box elements, allowing DBP to activate transcription and E4BP4 to antagonize DBP-mediated transcription (Supplementary Fig. 3a). In the Cry-null adrenal gland the expression of $D b p$ was constitutively elevated, while E4bp4 remained expressed at intermediate levels (Supplementary Fig. 3b). The constitutively high expression of $D b p$ represents a feature commonly observed for E-box-controlled genes (Supplementary Fig. 3c,d). Given our previous studies, showing E-box-dependent transcription of $D b p$ (refs. 18,20), these data favor a scenario in which the DBP-dependent constitutive activation directs the imbalanced super-expression of Hsd3b6 (see model in Supplementary Fig. 3d). It should be noted that although the transcriptional regulation of $H s d 3 b 6$ by CRY seems indirect through DBP/E4BP4, it can not be excluded that the activity of the Hsd3b6 promoter might be directly inhibited by CRY in vivo. The latter issue will have to be addressed by a chromatin immunoprecipitation assay using the adrenal ZG cell. 


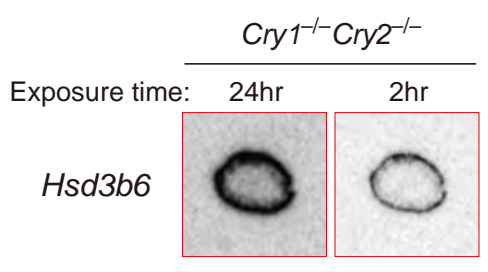

Supplementary Figure 4 Radioisotopic in situ hybridization analysis of Hsd3b6 in the Cry-null adrenal gland. Shown are in situ hybridization micrographs obtained at different exposure times. Shorter exposure results in reduced halation of strong Hsd3b6-positive signals that came from the outer layer of the adrenal cortex. 
a

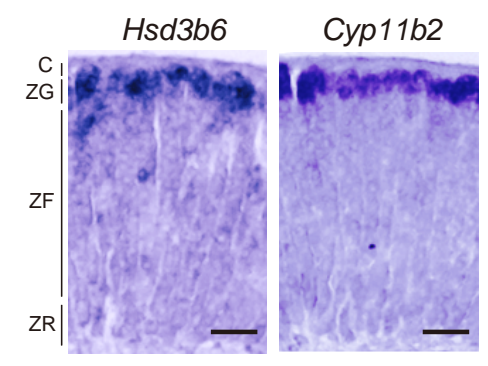

b

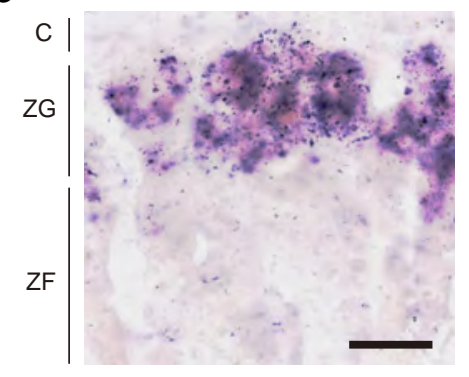

Supplementary Figure 5 Expression of Hsd3b6 and Cyp11b2 in the Cry-null adrenal ZG cells. (a) Digoxigenin in situ hybridization analysis of Hsd3b6 and Cyp11b2 in the Cry-null adrenal gland collected at CT0. The layered histoarchitecture of the adrenal: capsule (C); zona glomerulosa (ZG); zona fasciculata (ZF); zona reticularis (ZR). Bar, $50 \mu \mathrm{m}$. (b) Double-labeling in situ hybridization of digoxigenin-labeled Hsd3b6 and radioisotope-labeled Cyp11b2 probes. Show is a high magnification view of the region containing ZG cells in the Cry-null adrenal section. Hsd3b6 mRNA-positive cells were colored blue by nitroblue tetraformazan, while Cyp11b2 mRNA signal was shown as isotope-hitted silver grains by emulsion autography. Bar, $25 \mu \mathrm{m}$ 


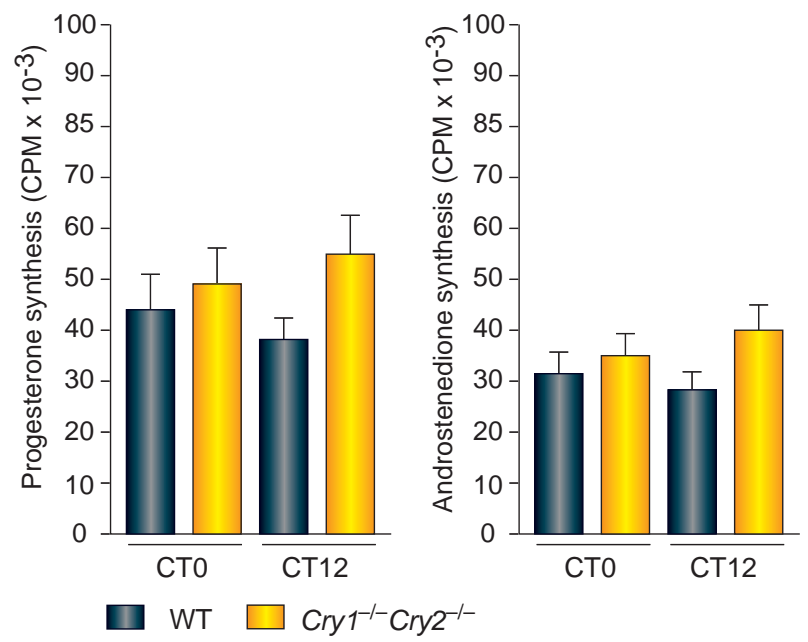

Supplementary Figure 6 3ß-HSD enzymatic activities in the decapsulated adrenal glands of WT and Cry-null mice at CT0 and CT12. The adrenal glands were mechanically separated into the capsular and decapsulated portions. The former portion

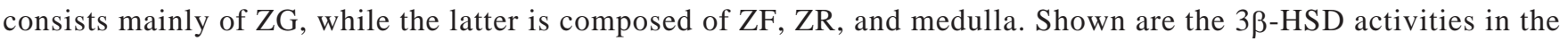
decapsulated portion of the adrenal glands. $3 \beta$-HSD activities were evaluated by progesterone synthesis from ${ }^{3} \mathrm{H}$-pregnenolone (left) and androstenedione synthesis from ${ }^{3} \mathrm{H}$-dehydroepiandrosterone (right). The synthesized steroid products were counted post fractionation by HPLC. Values are mean \pm s.e.m. $(n=10-12$, each). Note that the $3 \beta$-HSD activities in the decapsulated portions of the Cry-null adrenal gland were almost equivalent to those of the WT adrenal gland, while the highly enhanced 3 $\beta$-HSD activities were observed for the ZG cell-containing capsular portions of the Cry-null adrenal gland (see Fig. 4b). 


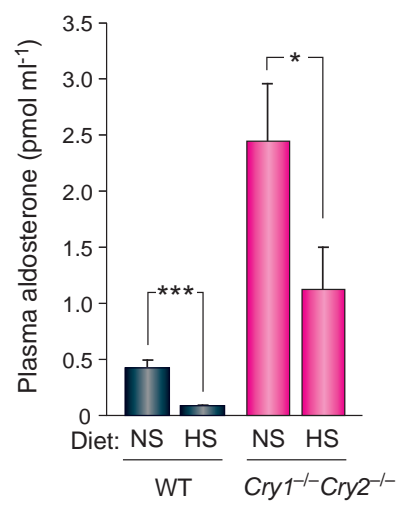

Supplementary Figure 7 Salt-dependent changes of plasma aldosterone levels in WT and Cry-null mice. Shown are the levels of PAC in WT and Cry-null mice at CT0 after 4-day loading on either a normal salt (NS) or a high salt (HS) diet ( $n=9-16$, each). Values are mean \pm s.e.m. $* P<0.05,{ }^{* * *} P<0.001$. Note that WT mice experienced a sharp reduction of PAC upon HS loading, while the response of the Cry-null mice was blunted, resulting in retention of excessive PAC even after HS loading. 
Supplementary Table 1 Subtype specification of $H s d 3 b$

\begin{tabular}{llll}
\hline $\begin{array}{l}\text { Isoform } \\
\text { type }\end{array}$ & $\begin{array}{l}\text { Amplicon } \\
\text { size }(\mathrm{bp})\end{array}$ & $\begin{array}{l}\text { Restriction } \\
\text { enzyme }\end{array}$ & $\begin{array}{l}\text { Digestion } \\
\text { pattern (bp) }\end{array}$ \\
\hline I & 629 & Accl & $455+174$ \\
II & 629 & HindIII & $508+121$ \\
III & 629 & BstBI & $439+190$ \\
IV and V & 629 & Avall & $335+187+107$ \\
VI & 629 & Ndel & $462+167$ \\
\hline
\end{tabular}

Supplementary Table 2 Subtype specification of Cyp11b

\begin{tabular}{llll}
\hline $\begin{array}{l}\text { Isoform } \\
\text { type }\end{array}$ & $\begin{array}{l}\text { Amplicon } \\
\text { size (bp) }\end{array}$ & $\begin{array}{l}\text { Restriction } \\
\text { enzyme }\end{array}$ & $\begin{array}{l}\text { Digestion } \\
\text { pattern (bp) }\end{array}$ \\
\hline I & 553 & Nhel & $401+152$ \\
II & 556 & Sacl & $390+99+67$ \\
\hline
\end{tabular}




\section{Supplementary Table 3 Overview of human and mouse Hsd3b genes}

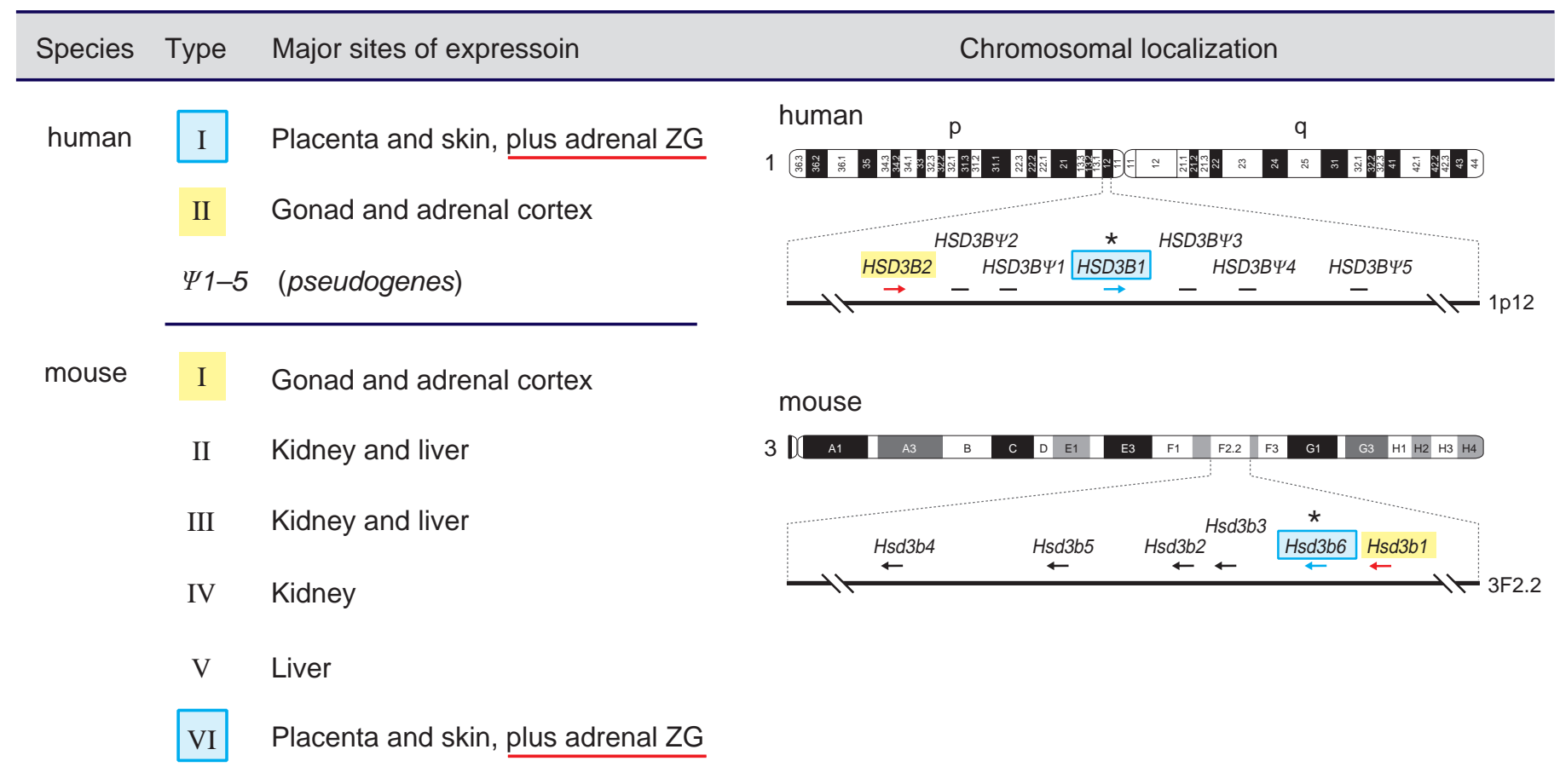

Chromosomal localization of the Hsd3b family: The Hsd3b genes in human and mouse form a syntenic cluster at chromosomal position $1 \mathrm{p} 12$ and 3F2.2, respectively. The human HSD3B region holds two expressed genes, HSD3B1 (blue arrow) and HSD3B2 (red arrow), and five pseudogenes, $\psi_{1-5}$ (black lines). On the other hand, the mouse $\mathrm{Hsd} 3 \mathrm{~b}$ region contains six expressed genes (types I to $\mathrm{VI}$ ). The mouse Hsd3b6 gene has been thought to encode a functional homolog of human HSD3B1 (see below). Note that the members of the mammalian Hsd3b family have been chronologically designated according to their order of elucidation in each species. Accordingly, the comparable gene names do not reflect functional similarities (refs 27,28). Information on human and mouse chromosomal DNA sequences were obtained from the Ensembl database (http://www.ensembl.org/index.html).

Major expression sites of HSD3Bs in human and mouse: The human HSD3B1 and mouse Hsd3b6 are functionally related because they are both expressed in placenta and skin (refs 27,28). Such expression profiles are unique, as all other $H s d 3 b$ isoforms are expressed in non-placental or non-dermal tissues. For example, the human HSD3B2 and mouse Hsd3b1 are expressed in gonad and adrenal cortex, forming another group that is distinct from the human HSD3B1 and mouse Hsd3b6. Moreover, as indicated by the red lines, we observed that human HSD3B1 and mouse Hsd3b6 genes are expressed in the ZG of the adrenal gland (see Figs 2, 3, 6). The subtype-specific tissue distribution profiles seem to be a conserved feature that is common to human and mouse HSD3b genes. 


\section{Supplementary Methods}

Plasma hormone analysis. Plasma aldosterone concentrations were measured using the Coat-A-Count Aldosteone kit (Siemens International.). This radioimmunoassay (RIA) differs from other available (commercial) assays in that it does not crossreact with any other steroid, including corticosterone. The plasma renin activity was determined according to a conventional RIA procedure (SRL, Tokyo, Japan), which measures the production of angiotensin I from the endogenous angiotensinogen for a 60 -min incubation at $37^{\circ} \mathrm{C}$.

Microarray analysis. Animals were sacrificed by cervical dislocation under a safety red light at the indicated time points in DD (see Fig. 2a). Total RNA was prepared from two pools of whole adrenal glands ( $n=6$, each pool) at each time point to duplicate our observations on two independent arrays. Total RNA was isolated with the RNeasy mini kit (Qiagen) and the integrity was assessed by analyzing aliquots on an Agilent 2100 Bioanalyzer (Agilent Technologies Japan). cDNA synthesis and cRNA labelling were performed using the One-Cycle Target Labelling and Control Reagents Kit (Affymetrix), and subsequent hybridization was performed with GeneChip Mouse Genome 4302.0 array (Affymetrix) according to the manufacture' protocol. For data analysis, we used the RMA (robust multi-array analysis) expression measure ${ }^{48}$ that represents the log transform of (background corrected and normalized) intensities of the GeneChips. The RMA measures were computed using the $\mathrm{R}$ package program, which is freely available on the web site (http://www.bioconductor.org). The value of each time point (Fig. 2a) was expressed as a mean of the two independent chips, which yielded equivalent results. For each transcript, values were normalized to the average expression level over the day in the WT adrenal gland. Genes analyzed in Fig. 2a were following: cytochrome P450, family 11, subfamily a, polypeptide 1 (Cyp11a1); cytochrome P450, family 11, subfamily b, polypeptide 2 (Cyp11b2); cytochrome P450, family 17, subfamily a, polypeptide 1 (Cyp17a1); cytochrome P450, family 19, subfamily a, polypeptide 1 (Cyp19a1), cytochrome P450, family 21, subfamily a, polypeptide 1 (Cyp21a1); hydroxysteroid 11-beta dehydrogenase 1 (Hsd11b1); hydroxysteroid (17-beta) dehydrogenase 1 (Hsd17b1); hydroxysteroid (17-beta) dehydrogenase 2 (Hsd17b2); hydroxysteroid (17-beta) dehydrogenase 3 (Hsd17b3); hydroxysteroid (17-beta) 
dehydrogenase 4 (Hsd17b4); hydroxysteroid (17-beta) dehydrogenase 6 (Hsd17b6); hydroxysteroid (17-beta) dehydrogenase 7 (Hsd17b7); hydroxysteroid (17-beta) dehydrogenase 12 (Hsd17b12); hydroxysteroid (17-beta) dehydrogenase 13 (Hsd17b13); hydroxy-delta-5-steroid dehydrogenase, 3 beta- and steroid delta-isomerase 1 (Hsd3b1); hydroxy-delta-5-steroid dehydrogenase, 3 beta- and steroid delta-isomerase 2 (Hsd3b2); hydroxy-delta-5-steroid dehydrogenase, 3 beta- and steroid delta-isomerase 3 (Hsd3b3); hydroxy-delta-5-steroid dehydrogenase, 3 beta- and steroid delta-isomerase 4 (Hsd3b4); hydroxy-delta-5-steroid dehydrogenase, 3 beta- and steroid delta-isomerase 5 (Hsd3b5); hydroxy-delta-5-steroid dehydrogenase, 3 beta- and steroid delta-isomerase 6 (Hsd3b6); hydroxy-delta-5-steroid dehydrogenase, 3 beta- and steroid delta-isomerase 7 (Hsd3b7); hydroxysteroid dehydrogenase like 1 (Hsdl1); hydroxysteroid dehydrogenase like 2 (Hsdl2); hydroxysteroid dehydrogenase like 2 (Hsdl2); NAD(P) dependent steroid dehydrogenase-like (Nsdhl); steroid 5 alpha-reductase 1 (Srd5a1); steroid 5 alpha-reductase 2 (Srd5a2); steroid 5 alpha-reductase 2-like 2 (Srd5a2l2); steroid 5 alpha-reductase 3 (Srd5a3); steroidogenic acute regulatory protein (Star); StAR-related lipid transfer (START) domain containing 4 (Stard4); StAR-related lipid transfer (START) domain containing 5 (Stard5); StAR-related lipid transfer (START) domain containing 6 (Stard6); steroid sulfatase (Sts).

Isoform specification of $\mathbf{H s d} 3 \boldsymbol{b}$ and Cyp11b. The gene isotypes of $H s d 3 b$ and $C y p 11 b$ were identified according to a previously established method ${ }^{39}$ with slight modifications. In brief, the amplification of $\mathrm{Hsd} 3 \mathrm{~b}$ cDNAs was done by PCR with the following primer set: 5'-cag acc atc cta gat gt-3' (Forward) and 5'-agg aag ctc aca gtt tcc a-3' (Reverse), yielding 629-bp DNA fragments from all known $H s d 3 b$ genes (sequence similarity enabled the amplification with a single PCR with a common primer set). For the amplification of Cyp11b cDNAs, we designed a primer set: 5'-ctg tca cca aaa gcc gtt c-3' (Forward) and 5'-ggt tcc gag cca gct ca-3' (Reverse), which can amplify the cDNA fragments of both Cyp11b1 and Cyp11b2. The primer sets used for $H s d 3 b$ and Cyp11b were designed to span introns to distinguish mRNA derived cDNA from amplified genomic DNA. The isotypes of the amplified genes were identified by digestion of the cDNA sequences with restriction enzymes unique to each of the isotypes. Supplementary Tables 1 and 2 summarize the restriction enzymes used and the resultant DNA digestion patterns. 
Quantitative RT-PCR. Adrenal total RNA was converted to cDNA using SuperScript III First-Strand Synthesis SuperMix (Invitrogen) and random hexamer primers. Quantitative PCR analysis of the individual cDNAs was achieved using the Platinum SYBR Green qPCR SuperMix-UDG (Invitrogen) with StepOnePlus real-time PCR monitoring system (Applied Biosystems). Results were normalized to Tbp mRNA levels. The primer sets used for the mouse adrenal study were following: Star (NM_011485), Fw: 5'-ccg gag cag agt ggt gtc a-3', Rv: 5'-gcc agt gga tga agc acc at-3'; Cyp11a1 (NM_019779), Fw: 5'-aag gta cag gag atg ctg cg-3', Rv: 5'-agt gtc tcc ttg atg ctg gc-3'; Hsd3b1 (NM_008293), Fw: 5'-agc atc cag aca ctc tca tc-3', Rv: 5'-gga gct ggt atg ata tag ggt a-3'; Hsd3b6 (NM_013821), Fw: 5'-cat cct tcc aca gtt cta gc-3', Rv: 5'-tgg tgt gag att aat gta ca-3'; Cyp21a1 (NM_009995), Fw: 5'-gct gtg gct ttc ctg ctt cac-3', Rv: 5'-ggc cca gct tga ggt cta act-3'; Cyp11b1 (NM_001033229), Fw: 5'-gtg agc cca tct tct gac ttt c-3', Rv: 5'-caa tgt gtc atg agt ggt cat ag-3'; Cyp11b2 (NM_009991), Fw: 5'-gtt ttc caa tgg tca ctc cag-3', Rv: 5'-gct tgc tgc ccc tac aaa c-3'; Tbp (NM_013684), Fw: 5'-atg gtg tgc aca gga gcc aag-3', Rv: 5'-tca tag cta ctg aac tgc tg-3'; 36B4 (AK010267), Fw: 5'-ctc act gag att cgg gat atg-3', Rv: 5'-ctc cca cct tgt ctc cag tc-3'. For the human adrenal study, we assayed human 36 b4 (NM_053275) using the following primer set: 5'-atg cag cag atc cgc atg t-3' (Fw) and 5'-ttg cgc atc atg gtg ttc tt-3' (Rv).

Promoter analysis of $\mathbf{H s d} \mathbf{3 b 6}$. Luciferase assays with ectopic expression of DBP and E4BP4 were carried out as described ${ }^{20}$ except that we used aldosterone-producing cell line H295R as a host. Reporter plasmids used were following: (i) Hsd3b6 promoter-luc: a 1128 bp genomic DNA fragment corresponding to the 5'-flanking region of mouse Hsd3b6 (-1128 to -1 from the transcription start site), cloned in pGL3-basic vector (Promega). (ii) M1-luc: Hsd3b6 promoterluc mutant in which the sequence of a putative D-box (distal site: -1069 to -1060) was changed from TGAGACGCTA to TTTATGTAAA. (iii) M2-luc: Hsd3b6 promoter-luc mutant in which the sequence of a putative D-box (proximal site: -1005 to -996) was changed from GTTAGGTATT to GGAGACGCTT. (iv) M1+M2-luc: Hsd3b6 promoter-luc mutant in which the distal and proximal D-box sites were both mutated as indicated in (ii) and (iii). Correct mutations were all verified by sequencing.

Radioisotopic in situ hybridization. The gene-specific probes used were as follows: for 
Hsd3b6, the anti-sense probe covering nucleotides 7-310 of the Hsd3b6 mRNA (Genbank, NM_013821); for Hsd3b1, nucleotides 22-360 (NM_008293); for Cyp11b1, nucleotides 1731-2304 (NM_001033229); for Cyp11b2, nucleotides 1744-2456 (S85260). The corresponding cDNA fragment was cloned and used as a template for the generation of riboprobes. The riboprobes were radiolabeled with [ $\left.{ }^{33} \mathrm{P}\right] \mathrm{UTP}$ (PerkinElmer), using a standard protocol for the cRNA synthesis. In situ hybridization was performed as described ${ }^{49}$. Briefly, paraformaldehyde fixed tissues were frozen and sectioned at a thickness of $30 \mu \mathrm{m}$. Then, the free-floating tissue sections were transferred through 4 x SSC, proteinase $\mathrm{K}\left(1 \mu \mathrm{g} \mathrm{ml}^{-1}, 0.1 \mathrm{M}\right.$ Tris buffer [pH 8.0]; $50 \mathrm{mM}$ EDTA) for $15 \mathrm{~min}$ at $37^{\circ} \mathrm{C}, 0.25 \%$ acetic anhydride in $0.1 \mathrm{M}$ triethanolamine for $10 \mathrm{~min}$, and 4 x SSC for $10 \mathrm{~min}$. The sections were then incubated in the hybridization buffer [55\% formamide, 10\% dextran sulfate, $10 \mathrm{mM}$ Tris-HCl (pH 8.0), $1 \mathrm{mM}$ EDTA (pH 8.0), 0.6 M NaCl, 0.2\% N-laurylsarcosine, $500 \mu \mathrm{g} \mathrm{ml}^{-1}$ tRNA, 1 x Denhardt's, $0.25 \%$ SDS, and $10 \mathrm{mM}$ dithiothreitol (DTT)] containing radiolabeled riboprobes for $16 \mathrm{~h}$ at $60^{\circ} \mathrm{C}$. Following a high-stringency posthybridization wash, the sections were treated with RNase A. Air-dried sections were exposed to X-ray films (Kodak Biomax).

Digoxigenin in situ hybridization. Since digoxigenin-labeled probes allow a better resolution than isotope probes for analyzing the cellular distribution of mRNA, we made digoxigenin-labeled antisense cRNA probes using digoxigenin-UTP (Roche Diagnostics) following a standard protocol of cRNA synthesis. Tissue preparation, prehybridization, hybridization, and posthybridization washing were the same as for isotope probe hybridization except that we used $20 \mu$ m-thick sections. The sections hybridized with the digoxigenin-labeled probe were processed for immunochemistry with the nucleic acid detection kit (Roche Diagnostics). Signals were visualized in a solution containing nitroblue tetrazolium salt (0.34 $\mathrm{mg} \mathrm{ml}^{-1}$ ) and 5-bromo-4-chloro-3-indolyl phosphate toluidinium salt (0.18 $\mathrm{mg} \mathrm{ml}^{-1}$ ) (Roche Diagnostics).

Double labeling histochemistry for Hsd3b6 and Cyp11b2. Immunocytochemistry of Hsd3b6 was performed using Hsd3b6-specific antibody that we raised in rabbit (see below for details). Free-floating sections were incubated for $12 \mathrm{~h}$ at room temperature with the affinity-purified Hsd3b6 antibody ( $3 \mathrm{ng} \mathrm{ml}^{-1}$ ), followed by incubation for $1 \mathrm{~h}$ at room temperature with donkey 
anti-rabbit IgG, conjugated to red fluorescent dye Alexafluor-594 (1:500 dilution; Invitrogen). The specificity was confirmed by dilution tests as well as signal absorption using the antigen peptide. To examine whether the Hsd3b6 is coexpressed with Cyp11b2 in the ZG cells, we performed double-labeling immunocytochemistry and in situ hybridization. Following the detection of Hsd3b6 immunofluorescence, sections were processed for the subsequent digoxigenin in situ hybridization for Cyp11b2. The prehybridization, hybridization, and posthybridization washes were as described above. The digoxigenin-labeled Cyp11b2 mRNA signals were stained blue with the nucleic acid detection kit (Roche Diagnostics).

Laser microdissection. Cryosections ( $5 \mu \mathrm{m}$ thick) of fresh-frozen adrenal glands were prepared using a cryostat (Leica) at $-17^{\circ} \mathrm{C}$ and mounted on POL-membrane slides (Leica). Sections were fixed for 2 min in an ice-cold mixture of ethanol and acetic acid (19:1), rinsed briefly in ice-cold water, stained for $1 \mathrm{~min}$ in ice-cold water containing $0.05 \%$ toluidine blue, followed by two brief washes in ice-cold water (all the solutions were RNase-free). After wiping off excess water, slides were quickly air dried for 1-2 min at room temperature. As soon as moistures in the sections decreased enough for laser-cutting, cells in the zona glomurosa and zona fasciculata were microdissected using a LMD6000 device (Leica; 40× magnification) and lysed into Trizol reagent (Invitrogen). Upon collection of $\sim 5000-10,000$ cells, total RNA was purified using the RNeasy micro kit (Qiagen), yielding on average $\sim 30$ ng of RNA. The ratio of 28S:18S rRNA in these RNA samples was approximately 2:1, thus confirming the integrity of the RNA isolated from laser-microdissected cells. Relative quantification of mRNA was done by quantitative RT-PCR using the comparative $\Delta \mathrm{Ct}$ method according to a standard protocol (http://docs.appliedbiosystems.com/pebiodocs/00105622. pdf).

Immunoblotting. Hsd3b6 polyclonal antibody was generated by immunizing rabbit with a synthetic peptide consisting of amino acid sequence 20-55 of the mouse Hsd3b6 protein, a region unique to the type VI isoform. The raised antibodies against Hsd3b6 were affinity-purified using the antigen peptide. A Cyp11a1 (P450scc) antibody was purchased from Chemicon (rabbit polyclonal, AB1244). Immunoblot analysis was performed using the following procedure. Adrenal glands, collected at either CT0 or CT12 ( $n=4$, each), were homogenized with a glass-Teflon homogenizer in $500 \mu \mathrm{l}$ of ice-cold $10 \mathrm{mM}$ Tris- $\mathrm{HCl}$ (pH 7.6) 
buffer, containing 0.25 M sucrose and a protease inhibitor cocktail (Roche). The insoluble debris and nuclei were pelleted by centrifugation at $800 \mathrm{~g}$ for $10 \mathrm{~min}$. Then, the supernatants were centrifuged at 105,000 g for 60 min (Optima MAX ultracentrifuge, Beckman) to sediment the fraction containing mitochondria and microsomes. The precipitates were denatured in Laemmli buffer post measuring the protein concentration by Bradford method. Western blotting was performed as previously described ${ }^{50}$. The proteins resolved on SDS-10\% polyacrylamide gel were transferred to a PVDF membrane. The imunoreactivities were visualized with enhanced chemiluminescence using horseradish peroxidase-conjugatged anti-rabbit IgG antibody (1:1000, GE healthcare).

Radiotelemetric blood pressure measurement. Arterial blood pressure (BP) was telemetrically monitored in conscious, freely moving animals with a battery-operated PA-C10 pressure transmitter (Data Sciences International, St Paul, MN). The transmitter was implanted by surgery under anesthesia with a mixture of ketamine (100 mg kg ${ }^{-1}$, ip) and xylazine (10 mg $\mathrm{kg}^{-1}$ ). The PA-C10 pressure-sensing catheter was inserted into the aortic arch through the left common carotid artery according to an established protocol ${ }^{51}$, and the PA-C10 rasiotransmitter body was placed in a subcutaneous pouch along the animal's right flank. Following surgery, animals were returned to their home cages and allowed to recover at least 1 week in LD before starting data collection in DD. Radio signals from the implanted PA-C10 were captured by RPC-1 receiver (Data Sciences International, St Paul, MN), and the data were online stored using the Dataquest ART data acquisition system (Data Sciences International, St Paul, MN). BP was monitored in 30-sec episodes at 5-min intervals.

48. Irizarry, R.A. et al. Summaries of Affymetrix GeneChip probe level data. Nucleic Acids Res 31, e15 (2003).

49. Shigeyoshi, Y. et al. Light-induced resetting of a mammalian circadian clock is associated with rapid induction of the mPer1 transcript. Cell 91, 1043-53 (1997).

50. Doi, M., Okano, T., Yujnovsky, I., Sassone-Corsi, P. \& Fukada, Y. Negative control of circadian clock regulator E4BP4 by casein kinase lepsilon-mediated phosphorylation. Curr Biol 14, 975-80 (2004).

51. Butz, G.M. \& Davisson, R.L. Long-term telemetric measurement of cardiovascular parameters in awake mice: a physiological genomics tool. Physiol Genomics 5, 89-97 (2001). 


\section{Supplementary Methods}

Plasma hormone analysis. Plasma aldosterone concentrations were measured using the Coat-A-Count Aldosteone kit (Siemens International.). This radioimmunoassay (RIA) differs from other available (commercial) assays in that it does not crossreact with any other steroid, including corticosterone. The plasma renin activity was determined according to a conventional RIA procedure (SRL, Tokyo, Japan), which measures the production of angiotensin I from the endogenous angiotensinogen for a 60 -min incubation at $37^{\circ} \mathrm{C}$.

Microarray analysis. Animals were sacrificed by cervical dislocation under a safety red light at the indicated time points in DD (see Fig. 2a). Total RNA was prepared from two pools of whole adrenal glands ( $n=6$, each pool) at each time point to duplicate our observations on two independent arrays. Total RNA was isolated with the RNeasy mini kit (Qiagen) and the integrity was assessed by analyzing aliquots on an Agilent 2100 Bioanalyzer (Agilent Technologies Japan). cDNA synthesis and cRNA labelling were performed using the One-Cycle Target Labelling and Control Reagents Kit (Affymetrix), and subsequent hybridization was performed with GeneChip Mouse Genome 4302.0 array (Affymetrix) according to the manufacture' protocol. For data analysis, we used the RMA (robust multi-array analysis) expression measure ${ }^{48}$ that represents the log transform of (background corrected and normalized) intensities of the GeneChips. The RMA measures were computed using the $\mathrm{R}$ package program, which is freely available on the web site (http://www.bioconductor.org). The value of each time point (Fig. 2a) was expressed as a mean of the two independent chips, which yielded equivalent results. For each transcript, values were normalized to the average expression level over the day in the WT adrenal gland. Genes analyzed in Fig. 2a were following: cytochrome P450, family 11, subfamily a, polypeptide 1 (Cyp11a1); cytochrome P450, family 11, subfamily b, polypeptide 2 (Cyp11b2); cytochrome P450, family 17, subfamily a, polypeptide 1 (Cyp17a1); cytochrome P450, family 19, subfamily a, polypeptide 1 (Cyp19a1), cytochrome P450, family 21, subfamily a, polypeptide 1 (Cyp21a1); hydroxysteroid 11-beta dehydrogenase 1 (Hsd11b1); hydroxysteroid (17-beta) dehydrogenase 1 (Hsd17b1); hydroxysteroid (17-beta) dehydrogenase 2 (Hsd17b2); hydroxysteroid (17-beta) dehydrogenase 3 (Hsd17b3); hydroxysteroid (17-beta) 
dehydrogenase 4 (Hsd17b4); hydroxysteroid (17-beta) dehydrogenase 6 (Hsd17b6); hydroxysteroid (17-beta) dehydrogenase 7 (Hsd17b7); hydroxysteroid (17-beta) dehydrogenase 12 (Hsd17b12); hydroxysteroid (17-beta) dehydrogenase 13 (Hsd17b13); hydroxy-delta-5-steroid dehydrogenase, 3 beta- and steroid delta-isomerase 1 (Hsd3b1); hydroxy-delta-5-steroid dehydrogenase, 3 beta- and steroid delta-isomerase 2 (Hsd3b2); hydroxy-delta-5-steroid dehydrogenase, 3 beta- and steroid delta-isomerase 3 (Hsd3b3); hydroxy-delta-5-steroid dehydrogenase, 3 beta- and steroid delta-isomerase 4 (Hsd3b4); hydroxy-delta-5-steroid dehydrogenase, 3 beta- and steroid delta-isomerase 5 (Hsd3b5); hydroxy-delta-5-steroid dehydrogenase, 3 beta- and steroid delta-isomerase 6 (Hsd3b6); hydroxy-delta-5-steroid dehydrogenase, 3 beta- and steroid delta-isomerase 7 (Hsd3b7); hydroxysteroid dehydrogenase like 1 (Hsdl1); hydroxysteroid dehydrogenase like 2 (Hsdl2); hydroxysteroid dehydrogenase like 2 (Hsdl2); NAD(P) dependent steroid dehydrogenase-like (Nsdhl); steroid 5 alpha-reductase 1 (Srd5a1); steroid 5 alpha-reductase 2 (Srd5a2); steroid 5 alpha-reductase 2-like 2 (Srd5a2l2); steroid 5 alpha-reductase 3 (Srd5a3); steroidogenic acute regulatory protein (Star); StAR-related lipid transfer (START) domain containing 4 (Stard4); StAR-related lipid transfer (START) domain containing 5 (Stard5); StAR-related lipid transfer (START) domain containing 6 (Stard6); steroid sulfatase (Sts).

Isoform specification of $\mathbf{H s d} 3 \boldsymbol{b}$ and Cyp11b. The gene isotypes of $H s d 3 b$ and $C y p 11 b$ were identified according to a previously established method ${ }^{39}$ with slight modifications. In brief, the amplification of $\mathrm{Hsd} 3 \mathrm{~b}$ cDNAs was done by PCR with the following primer set: 5'-cag acc atc cta gat gt-3' (Forward) and 5'-agg aag ctc aca gtt tcc a-3' (Reverse), yielding 629-bp DNA fragments from all known $H s d 3 b$ genes (sequence similarity enabled the amplification with a single PCR with a common primer set). For the amplification of Cyp11b cDNAs, we designed a primer set: 5'-ctg tca cca aaa gcc gtt c-3' (Forward) and 5'-ggt tcc gag cca gct ca-3' (Reverse), which can amplify the cDNA fragments of both Cyp11b1 and Cyp11b2. The primer sets used for $H s d 3 b$ and Cyp11b were designed to span introns to distinguish mRNA derived cDNA from amplified genomic DNA. The isotypes of the amplified genes were identified by digestion of the cDNA sequences with restriction enzymes unique to each of the isotypes. Supplementary Tables 1 and 2 summarize the restriction enzymes used and the resultant DNA digestion patterns. 
Quantitative RT-PCR. Adrenal total RNA was converted to cDNA using SuperScript III First-Strand Synthesis SuperMix (Invitrogen) and random hexamer primers. Quantitative PCR analysis of the individual cDNAs was achieved using the Platinum SYBR Green qPCR SuperMix-UDG (Invitrogen) with StepOnePlus real-time PCR monitoring system (Applied Biosystems). Results were normalized to Tbp mRNA levels. The primer sets used for the mouse adrenal study were following: Star (NM_011485), Fw: 5'-ccg gag cag agt ggt gtc a-3', Rv: 5'-gcc agt gga tga agc acc at-3'; Cyp11a1 (NM_019779), Fw: 5'-aag gta cag gag atg ctg cg-3', Rv: 5'-agt gtc tcc ttg atg ctg gc-3'; Hsd3b1 (NM_008293), Fw: 5'-agc atc cag aca ctc tca tc-3', Rv: 5'-gga gct ggt atg ata tag ggt a-3'; Hsd3b6 (NM_013821), Fw: 5'-cat cct tcc aca gtt cta gc-3', Rv: 5'-tgg tgt gag att aat gta ca-3'; Cyp21a1 (NM_009995), Fw: 5'-gct gtg gct ttc ctg ctt cac-3', Rv: 5'-ggc cca gct tga ggt cta act-3'; Cyp11b1 (NM_001033229), Fw: 5'-gtg agc cca tct tct gac ttt c-3', Rv: 5'-caa tgt gtc atg agt ggt cat ag-3'; Cyp11b2 (NM_009991), Fw: 5'-gtt ttc caa tgg tca ctc cag-3', Rv: 5'-gct tgc tgc ccc tac aaa c-3'; Tbp (NM_013684), Fw: 5'-atg gtg tgc aca gga gcc aag-3', Rv: 5'-tca tag cta ctg aac tgc tg-3'; 36B4 (AK010267), Fw: 5'-ctc act gag att cgg gat atg-3', Rv: 5'-ctc cca cct tgt ctc cag tc-3'. For the human adrenal study, we assayed human 36 b4 (NM_053275) using the following primer set: 5'-atg cag cag atc cgc atg t-3' (Fw) and 5'-ttg cgc atc atg gtg ttc tt-3' (Rv).

Promoter analysis of $\mathbf{H s d} \mathbf{3 b 6}$. Luciferase assays with ectopic expression of DBP and E4BP4 were carried out as described ${ }^{20}$ except that we used aldosterone-producing cell line H295R as a host. Reporter plasmids used were following: (i) Hsd3b6 promoter-luc: a 1128 bp genomic DNA fragment corresponding to the 5'-flanking region of mouse Hsd3b6 (-1128 to -1 from the transcription start site), cloned in pGL3-basic vector (Promega). (ii) M1-luc: Hsd3b6 promoterluc mutant in which the sequence of a putative D-box (distal site: -1069 to -1060) was changed from TGAGACGCTA to TTTATGTAAA. (iii) M2-luc: Hsd3b6 promoter-luc mutant in which the sequence of a putative D-box (proximal site: -1005 to -996) was changed from GTTAGGTATT to GGAGACGCTT. (iv) M1+M2-luc: Hsd3b6 promoter-luc mutant in which the distal and proximal D-box sites were both mutated as indicated in (ii) and (iii). Correct mutations were all verified by sequencing.

Radioisotopic in situ hybridization. The gene-specific probes used were as follows: for 
Hsd3b6, the anti-sense probe covering nucleotides 7-310 of the Hsd3b6 mRNA (Genbank, NM_013821); for Hsd3b1, nucleotides 22-360 (NM_008293); for Cyp11b1, nucleotides 1731-2304 (NM_001033229); for Cyp11b2, nucleotides 1744-2456 (S85260). The corresponding cDNA fragment was cloned and used as a template for the generation of riboprobes. The riboprobes were radiolabeled with [ $\left.{ }^{33} \mathrm{P}\right] \mathrm{UTP}$ (PerkinElmer), using a standard protocol for the cRNA synthesis. In situ hybridization was performed as described ${ }^{49}$. Briefly, paraformaldehyde fixed tissues were frozen and sectioned at a thickness of $30 \mu \mathrm{m}$. Then, the free-floating tissue sections were transferred through 4 x SSC, proteinase $\mathrm{K}\left(1 \mu \mathrm{g} \mathrm{ml}^{-1}, 0.1 \mathrm{M}\right.$ Tris buffer [pH 8.0]; $50 \mathrm{mM}$ EDTA) for $15 \mathrm{~min}$ at $37^{\circ} \mathrm{C}, 0.25 \%$ acetic anhydride in $0.1 \mathrm{M}$ triethanolamine for $10 \mathrm{~min}$, and 4 x SSC for $10 \mathrm{~min}$. The sections were then incubated in the hybridization buffer [55\% formamide, 10\% dextran sulfate, $10 \mathrm{mM}$ Tris-HCl (pH 8.0), $1 \mathrm{mM}$ EDTA (pH 8.0), 0.6 M NaCl, 0.2\% N-laurylsarcosine, $500 \mu \mathrm{g} \mathrm{ml}^{-1}$ tRNA, 1 x Denhardt's, $0.25 \%$ SDS, and $10 \mathrm{mM}$ dithiothreitol (DTT)] containing radiolabeled riboprobes for $16 \mathrm{~h}$ at $60^{\circ} \mathrm{C}$. Following a high-stringency posthybridization wash, the sections were treated with RNase A. Air-dried sections were exposed to X-ray films (Kodak Biomax).

Digoxigenin in situ hybridization. Since digoxigenin-labeled probes allow a better resolution than isotope probes for analyzing the cellular distribution of mRNA, we made digoxigenin-labeled antisense cRNA probes using digoxigenin-UTP (Roche Diagnostics) following a standard protocol of cRNA synthesis. Tissue preparation, prehybridization, hybridization, and posthybridization washing were the same as for isotope probe hybridization except that we used $20 \mu$ m-thick sections. The sections hybridized with the digoxigenin-labeled probe were processed for immunochemistry with the nucleic acid detection kit (Roche Diagnostics). Signals were visualized in a solution containing nitroblue tetrazolium salt (0.34 $\mathrm{mg} \mathrm{ml}^{-1}$ ) and 5-bromo-4-chloro-3-indolyl phosphate toluidinium salt (0.18 $\mathrm{mg} \mathrm{ml}^{-1}$ ) (Roche Diagnostics).

Double labeling histochemistry for Hsd3b6 and Cyp11b2. Immunocytochemistry of Hsd3b6 was performed using Hsd3b6-specific antibody that we raised in rabbit (see below for details). Free-floating sections were incubated for $12 \mathrm{~h}$ at room temperature with the affinity-purified Hsd3b6 antibody ( $3 \mathrm{ng} \mathrm{ml}^{-1}$ ), followed by incubation for $1 \mathrm{~h}$ at room temperature with donkey 
anti-rabbit IgG, conjugated to red fluorescent dye Alexafluor-594 (1:500 dilution; Invitrogen). The specificity was confirmed by dilution tests as well as signal absorption using the antigen peptide. To examine whether the Hsd3b6 is coexpressed with Cyp11b2 in the ZG cells, we performed double-labeling immunocytochemistry and in situ hybridization. Following the detection of Hsd3b6 immunofluorescence, sections were processed for the subsequent digoxigenin in situ hybridization for Cyp11b2. The prehybridization, hybridization, and posthybridization washes were as described above. The digoxigenin-labeled Cyp11b2 mRNA signals were stained blue with the nucleic acid detection kit (Roche Diagnostics).

Laser microdissection. Cryosections ( $5 \mu \mathrm{m}$ thick) of fresh-frozen adrenal glands were prepared using a cryostat (Leica) at $-17^{\circ} \mathrm{C}$ and mounted on POL-membrane slides (Leica). Sections were fixed for 2 min in an ice-cold mixture of ethanol and acetic acid (19:1), rinsed briefly in ice-cold water, stained for $1 \mathrm{~min}$ in ice-cold water containing $0.05 \%$ toluidine blue, followed by two brief washes in ice-cold water (all the solutions were RNase-free). After wiping off excess water, slides were quickly air dried for 1-2 min at room temperature. As soon as moistures in the sections decreased enough for laser-cutting, cells in the zona glomurosa and zona fasciculata were microdissected using a LMD6000 device (Leica; 40× magnification) and lysed into Trizol reagent (Invitrogen). Upon collection of $\sim 5000-10,000$ cells, total RNA was purified using the RNeasy micro kit (Qiagen), yielding on average $\sim 30$ ng of RNA. The ratio of 28S:18S rRNA in these RNA samples was approximately 2:1, thus confirming the integrity of the RNA isolated from laser-microdissected cells. Relative quantification of mRNA was done by quantitative RT-PCR using the comparative $\Delta \mathrm{Ct}$ method according to a standard protocol (http://docs.appliedbiosystems.com/pebiodocs/00105622. pdf).

Immunoblotting. Hsd3b6 polyclonal antibody was generated by immunizing rabbit with a synthetic peptide consisting of amino acid sequence 20-55 of the mouse Hsd3b6 protein, a region unique to the type VI isoform. The raised antibodies against Hsd3b6 were affinity-purified using the antigen peptide. A Cyp11a1 (P450scc) antibody was purchased from Chemicon (rabbit polyclonal, AB1244). Immunoblot analysis was performed using the following procedure. Adrenal glands, collected at either CT0 or CT12 ( $n=4$, each), were homogenized with a glass-Teflon homogenizer in $500 \mu \mathrm{l}$ of ice-cold $10 \mathrm{mM}$ Tris- $\mathrm{HCl}$ (pH 7.6) 
buffer, containing 0.25 M sucrose and a protease inhibitor cocktail (Roche). The insoluble debris and nuclei were pelleted by centrifugation at $800 \mathrm{~g}$ for $10 \mathrm{~min}$. Then, the supernatants were centrifuged at 105,000 g for 60 min (Optima MAX ultracentrifuge, Beckman) to sediment the fraction containing mitochondria and microsomes. The precipitates were denatured in Laemmli buffer post measuring the protein concentration by Bradford method. Western blotting was performed as previously described ${ }^{50}$. The proteins resolved on SDS-10\% polyacrylamide gel were transferred to a PVDF membrane. The imunoreactivities were visualized with enhanced chemiluminescence using horseradish peroxidase-conjugatged anti-rabbit IgG antibody (1:1000, GE healthcare).

Radiotelemetric blood pressure measurement. Arterial blood pressure (BP) was telemetrically monitored in conscious, freely moving animals with a battery-operated PA-C10 pressure transmitter (Data Sciences International, St Paul, MN). The transmitter was implanted by surgery under anesthesia with a mixture of ketamine (100 mg kg ${ }^{-1}$, ip) and xylazine (10 mg $\mathrm{kg}^{-1}$ ). The PA-C10 pressure-sensing catheter was inserted into the aortic arch through the left common carotid artery according to an established protocol ${ }^{51}$, and the PA-C10 rasiotransmitter body was placed in a subcutaneous pouch along the animal's right flank. Following surgery, animals were returned to their home cages and allowed to recover at least 1 week in LD before starting data collection in DD. Radio signals from the implanted PA-C10 were captured by RPC-1 receiver (Data Sciences International, St Paul, MN), and the data were online stored using the Dataquest ART data acquisition system (Data Sciences International, St Paul, MN). BP was monitored in 30-sec episodes at 5-min intervals.

48. Irizarry, R.A. et al. Summaries of Affymetrix GeneChip probe level data. Nucleic Acids Res 31, e15 (2003).

49. Shigeyoshi, Y. et al. Light-induced resetting of a mammalian circadian clock is associated with rapid induction of the mPer1 transcript. Cell 91, 1043-53 (1997).

50. Doi, M., Okano, T., Yujnovsky, I., Sassone-Corsi, P. \& Fukada, Y. Negative control of circadian clock regulator E4BP4 by casein kinase lepsilon-mediated phosphorylation. Curr Biol 14, 975-80 (2004).

51. Butz, G.M. \& Davisson, R.L. Long-term telemetric measurement of cardiovascular parameters in awake mice: a physiological genomics tool. Physiol Genomics 5, 89-97 (2001). 